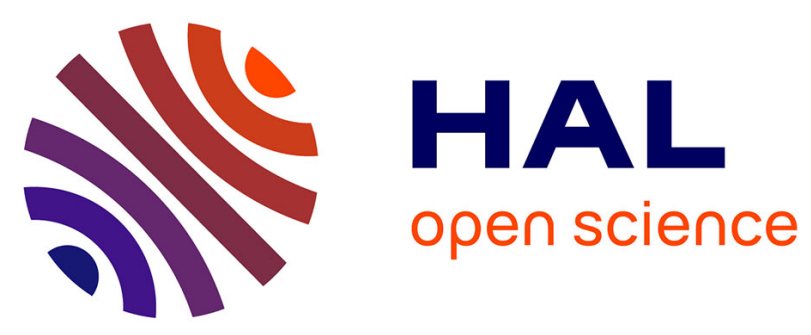

\title{
Lagrangian-Antidiffusive Remap schemes for non-local multi-class traffic flow models
}

Felisia Angela Chiarello, Paola Goatin, Luis Miguel Villada

\section{To cite this version:}

Felisia Angela Chiarello, Paola Goatin, Luis Miguel Villada. Lagrangian-Antidiffusive Remap schemes for non-local multi-class traffic flow models. Computational \& Applied Mathematics, 2020, 39 (60), 10.1007/s40314-020-1097-9 . hal-01952378v2

\section{HAL Id: hal-01952378 \\ https://hal.science/hal-01952378v2}

Submitted on 2 Dec 2019

HAL is a multi-disciplinary open access archive for the deposit and dissemination of scientific research documents, whether they are published or not. The documents may come from teaching and research institutions in France or abroad, or from public or private research centers.
L'archive ouverte pluridisciplinaire HAL, est destinée au dépôt et à la diffusion de documents scientifiques de niveau recherche, publiés ou non, émanant des établissements d'enseignement et de recherche français ou étrangers, des laboratoires publics ou privés. 


\title{
Lagrangian-Antidiffusive Remap schemes for non-local multi-class traffic flow models
}

\author{
Felisia Angela Chiarello ${ }^{1} \quad$ Paola Goatin $^{1} \quad$ Luis Miguel Villada $^{2}$
}

December 2, 2019

\begin{abstract}
This paper focuses on the numerical approximation of the solutions of a class of nonlocal systems in one space dimension, arising in traffic modeling. We propose alternative simple schemes by splitting the non-local conservation laws into two different equations, namely, the Lagrangian and the remap steps. We provide some properties and estimates recovered by approximating the problem with the Lagrangian-Antidiffusive Remap (LAR) scheme, and we prove the convergence to weak solutions in the scalar case. Finally, we show some numerical simulations illustrating the efficiency of the L-AR schemes in comparison with classical first and second-order numerical schemes.
\end{abstract}

\section{Introduction}

The aim of this paper is to propose an efficient and easy-to-implement numerical scheme to compute approximate solutions of the non-local multi-class traffic flow model introduced in [10]. It consists in the following $M \times M$ system of conservation laws with non-local velocities

$$
\partial_{t} \rho_{i}(t, x)+\partial_{x}\left(\rho_{i}(t, x) v_{i}\left(\left(r * \omega_{i}\right)(t, x)\right)\right)=0, \quad i=1, \ldots, M,
$$

where we set

$$
\begin{aligned}
& r(t, x):=\sum_{i=1}^{M} \rho_{i}(t, x), \quad v_{i}(\xi):=v_{i}^{\max } \psi(\xi), \\
& \left(r * \omega_{i}\right)(t, x):=\int_{x}^{x+\eta_{i}} r(t, y) \omega_{i}(y-x) \mathrm{d} y, \\
& \rho_{i}(0, x)=\rho_{i}^{0}(x), \quad i=1, \ldots, M .
\end{aligned}
$$

We make the following hypotheses:

$$
\omega_{i} \in \mathbf{C}^{\mathbf{1}}\left(\left[0, \eta_{i}\right] ; \mathbb{R}^{+}\right), \eta_{i}>0, \text { s.t. } \omega_{i}^{\prime} \leq 0, \int_{0}^{\eta_{i}} \omega_{i}(y) \mathrm{d} y=J_{i} \text { and } W_{0}:=\max _{i=1, \ldots, M} \omega_{i}(0) ;
$$

(H) $0<v_{1}^{\max } \leq v_{2}^{\max } \leq \ldots \leq v_{M}^{\max }$

$\psi: \mathbb{R}^{+} \rightarrow \mathbb{R}^{+}$is a smooth non-increasing function s.t. $\psi(0)=1, \psi(r)=0$ for $r \geq 1$.

\footnotetext{
${ }^{1}$ Inria Sophia Antipolis - Méditerranée, Université Côte d'Azur, Inria, CNRS, LJAD, 2004 route des Lucioles - BP 93, 06902 Sophia Antipolis Cedex, France. E-mail: \{felisia.chiarello, paola.goatin\}@inria.fr

${ }^{2}$ GIMNAP-Departamento de Matemáticas, Universidad del Bío-Bío, Casilla 5-C, Concepción, Chile and $\mathrm{CI}^{2} \mathrm{MA}$, Universidad de Concepción, Casilla 160-C, Concepción, Chile. E-mail: lvillada@ubiobio.cl
} 
From the application point of view, $\rho_{i}$ represents the density of the $i$-th vehicle class, characterized by its maximal velocity $v_{i}^{\max }$ and its interaction kernel $\omega_{i}$, which accounts for the reaction to downstream traffic distribution. In particular, $\eta_{i}$ is proportional to the look-ahead distance of drivers and $J_{i}$ represents the interaction strength. Equations (1.1) are coupled through the velocity function, which depends on an integral evaluation of the total traffic density $r$. For simplicity, in this work we will consider linear decreasing velocities setting $\psi(r)=\max \{1-r, 0\}$.

Model (1.1) is a multi-class extension of the one-dimensional scalar conservation law with non-local flux proposed in [3, 15. It can also be seen as the non-local generalization of the "local" multi-population model for traffic flow described in [2. Indeed, one of the limitations of the standard Lighthill-Whitham-Richards (LWR) traffic flow model [17, 18] is the first in first out rule, conversely in multi-class dynamic faster vehicles can overtake slower ones and slower vehicles slow down the faster ones. In our setting, the non-local dependence of the speed functions $v_{i}$ describes the reaction of drivers that adapt their velocity with respect to what happens in front of them in terms of downstream traffic, assigning greater importance to closer vehicles, see also 9 .

Since solutions to (1.1), 1.4 may be discontinuous, they are intended in the following weak sense.

Definition 1. A function $\boldsymbol{\rho}=\left(\rho_{1}, \ldots, \rho_{M}\right) \in\left(\mathbf{L}^{\mathbf{1}} \cap \mathbf{L}^{\infty}\right)\left(\left[0, T\left[\times \mathbb{R} ; \mathbb{R}^{M}\right), T>0\right.\right.$, is a weak solution of (1.1), (1.4) if

$$
\int_{0}^{T} \int_{-\infty}^{\infty}\left(\rho_{i} \partial_{t} \varphi+\rho_{i} v_{i}\left(r * \omega_{i}\right) \partial_{x} \varphi\right)(t, x) \mathrm{d} x \mathrm{~d} t+\int_{-\infty}^{\infty} \rho_{i}^{0}(x) \varphi(0, x) \mathrm{d} x=0
$$

for all $\varphi \in \mathbf{C}_{c}^{1}(]-\infty, T[\times \mathbb{R} ; \mathbb{R}), i=1, \ldots, M$.

The existence of solutions, locally in time, was proved in [10] by constructing a sequence of approximate solutions via an up-wind finite volume scheme, and proving its convergence. We observe that, due to the lack of smoothness of the convolutions kernels $\omega_{i}$ on $\mathbb{R}$, the general well-posedness results given in 11 cannot be applied.

In this work, we focus on the numerical aspects. We remark that the computation of numerical solutions for (1.1) is challenging due to the high non-linearity of the system and the dependence of the flux function on convolution terms, which increase the computational cost. For these reasons, the development of efficient numerical strategies is crucial to precisely capture solutions' behaviour. The first-order schemes used in [9, 10, are known to be very diffusive. On the other hand, high-order Discontinuous Galerkin and Finite Volume WENO schemes, which are based on quadratic polynomial reconstruction in each cell to evaluate the non-local terms in order to obtain high-order of accuracy, were constructed and tested for the scalar cases in [8] and extended to multi-class cases in [11].

The aim of this paper is to present a generalization to non-local systems of the LagrangianAntidiffusive Remap (L-AR) schemes introduced in [5, 6, in order to compute approximate solutions of model (1.1). This type of schemes are constructed exploiting the concentrationtimes-velocity form of the fluxes in (1.1). In [5], one step L-AR schemes were applied to multi-class Lighthill-Whitham-Richards (MCLWR) traffic models and they were extended to polydisperse sedimentation models in [6]. L-AR schemes do not rely on spectral (characteristics) information and their implementation is as easy as that one of first- and second-order of accuracy schemes introduced in [7]. These L-AR schemes are supported by a partial analysis 
for $M=1$, with the conclusion that under a suitable CFL condition, these schemes converge to a weak solution. Numerical experiments show that L-AR schemes perform better in terms of resolution, accuracy and efficiency, especially for large values of $M$.

The paper is organized as follows. Section 2 presents the L-AR schemes. We recover some properties of the schemes in both scalar and multi-class cases. In the scalar case, we obtain uniform $\mathbf{L}^{\infty}, \mathbf{B V}$ estimates on the approximate solutions computed through the L-AR schemes, which provide an alternative proof of existence of weak solutions. In Section 3, we recall classical first-order schemes used to approximate the solutions of the non-local problem (1.1) and we provide a second-order version of a Godunov type numerical scheme. Finally, in Section 4, we present some numerical simulations, analyzing the $\mathbf{L}^{\mathbf{1}}$-error of the approximate solutions of (1.1) computed with different schemes and considering smooth and discontinuous initial data.

\section{Lagrangian-Antidiffusive Remap (L-AR) schemes}

\subsection{Discretization}

First of all, we extend $\omega_{i}(x)=0$ for $x>\eta_{i}$. For $j \in \mathbb{Z}$ and $n \in \mathbb{N}$, let $x_{j+1 / 2}=j \Delta x$ be the cells interfaces, $x_{j}=(j-1 / 2) \Delta x$ the cells centers and $t^{n}=n \Delta t$ the time mesh. In the paper, we will set $\lambda=\frac{\Delta t}{\Delta x}$. We aim at constructing a finite volume approximate solution $\rho^{\Delta x}=\left(\rho_{1}^{\Delta x}, \ldots, \rho_{M}^{\Delta x}\right)$, with $\rho_{i}^{\Delta x}(t, x)=\rho_{i, j}^{n}$ for $(t, x) \in C_{j}^{n}=\left[t^{n}, t^{n+1}[\times] x_{j-1 / 2}, x_{j+1 / 2}\right]$ and $i=1, \ldots, M$.

To this end, we approximate the initial datum $\rho_{i}^{0}$ for $i=1, \ldots, M$ with a piece-wise constant function

$$
\rho_{i, j}^{0}=\frac{1}{\Delta x} \int_{x_{j-1 / 2}}^{x_{j+1 / 2}} \rho_{i}^{0}(x) \mathrm{d} x, \quad j \in \mathbb{Z} .
$$

Similarly, for the kernel, we set

$$
\omega_{i}^{k}:=\frac{1}{\Delta x} \int_{(k-1) \Delta x}^{k \Delta x} \omega_{i}(x) \mathrm{d} x, \quad k \in \mathbb{N}^{*},
$$

so that $\Delta x \sum_{k=1}^{+\infty} \omega_{i}^{k}=\int_{0}^{\eta_{i}} \omega_{i}(x) \mathrm{d} x=J_{i}$ (the sum is indeed finite since $\omega_{i}^{k}=0$ for $k \geq N_{i}$ sufficiently large). Moreover, we set $r_{j+k}^{n}=\sum_{i=1}^{M} \rho_{i, j+k}^{n}$ for $k \in \mathbb{N}$ and

$$
V_{i, j+1 / 2}^{n}:=v_{i}^{\max } \psi\left(\Delta x \sum_{k=1}^{+\infty} \omega_{i}^{k} r_{j+k}^{n}\right), \quad i=1, \ldots, M, \quad j \in \mathbb{Z} .
$$

We formally rewrite 1.1 as

$$
\partial_{t} \rho_{i}+\rho_{i} \partial_{x}\left(v_{i}\left(r * \omega_{i}\right)\right)+v_{i}\left(r * \omega_{i}\right) \partial_{x} \rho_{i}=0, \quad i=1, \ldots, M .
$$

L-AR schemes are obtained splitting (2.4) into two different equations, which are solved successively for each time iteration. To advance the solution from time $t$ to $t+\Delta t$, we first apply a Lagrangian method [16] to solve

$$
\partial_{t} \rho_{i}+\rho_{i} \partial_{x}\left(v_{i}\left(r * \omega_{i}\right)\right)=0
$$


and we use this solution, evolved over a time interval of length $\Delta t$, as initial condition for solving in a second step the transport equation [4]

$$
\partial_{t} \rho_{i}+v_{i}\left(r * \omega_{i}\right) \partial_{x} \rho_{i}=0
$$

whose solution, again evolved over a time interval of length $\Delta t$, provides the sought approximate solution of (1.1) valid for $t+\Delta t$.

\subsection{Discretization of the Lagrangian step}

We observe that, defining $\tau_{i}:=1 / \rho_{i}$, one obtains from (2.5) the conservation mass equation in Lagrangian coordinates

$$
\rho_{i} \partial_{t} \tau_{i}-\partial_{x}\left(v_{i}\left(r * \omega_{i}\right)\right)=0 .
$$

In other words, solving (2.5), or equivalently (2.7), means solving the original equation (1.1)

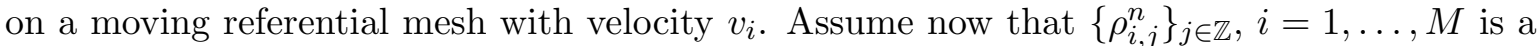
approximate solution of (1.1) in the sense of finite volume methods (2.1)-(2.3) at time $t=t^{n}$, then a numerical solution $\left\{\rho_{i, j}^{n+1,-}\right\}_{j \in \mathbb{Z}}$ of the equation 2.7 at time $\Delta t$ can be naturally computed by

$$
\rho_{i, j}^{n+1,-}\left[\Delta x+\left(V_{i, j+1 / 2}^{n}-V_{i, j-1 / 2}^{n}\right) \Delta t\right]=\Delta x \rho_{i, j}^{n}, \quad i=1, \ldots, M, \quad j \in \mathbb{Z},
$$

since (2.8) expresses that the initial mass in the cell $\left[x_{j-1 / 2}, x_{j+1 / 2}\right]$ at time $t^{n}$ equals the mass in the modified cell $\left[\bar{x}_{j-1 / 2}, \bar{x}_{j+1 / 2}\right]$ at time $\Delta t$, where $\bar{x}_{j+1 / 2}=x_{j+1 / 2}+V_{i, j+1 / 2}^{n} \Delta t$ are the new interface positions for all $j$. We have the following properties.

Lemma 1. Assume that the time step satisfies the following condition:

$$
\Delta t \leq\left(v_{M}^{\max }\left\|\psi^{\prime}\right\|_{\infty}\left\|r^{n}\right\|_{\infty} W_{0}\right)^{-1} .
$$

If $\left\{\boldsymbol{\rho}_{j}^{n+1,-}\right\}_{j \in \mathbb{Z}}$ denotes the numerical solution computed by the scheme (2.8), then the following bounds hold:

(i) If $\rho_{i, j}^{n} \geq 0$ for all $j \in \mathbb{Z}$, then $\rho_{i, j}^{n+1,-} \geq 0$ for all $j \in \mathbb{Z}$.

(ii) In the scalar case $M=1$, the following maximum property holds:

$$
\min \left\{\rho_{j}^{n}, \ldots, \rho_{j+N}^{n}\right\} \leq \rho_{j}^{n+1,-} \leq \max \left\{\rho_{j}^{n}, \ldots, \rho_{j+N}^{n}\right\} \quad \forall j \in \mathbb{Z} .
$$

Proof. (i) Suppose that $\rho_{i, j}^{n} \geq 0$ for all $j \in \mathbb{Z}$ and $i=1, \ldots, M$. From (2.8) we have

$$
\rho_{i, j}^{n+1,-}=\frac{\rho_{i, j}^{n}}{1+\lambda\left(V_{i, j+1 / 2}^{n}-V_{i, j-1 / 2}^{n}\right)} .
$$

If $V_{i, j+1 / 2}^{n} \geq V_{i, j-1 / 2}^{n}$ then it is clear that $\rho_{i, j}^{n+1,-} \geq 0$. Consider now the case $V_{i, j+1 / 2}^{n} \leq$ $V_{i, j-1 / 2}^{n}$. We can compute

$$
V_{i, j-1 / 2}^{n}-V_{i, j+1 / 2}^{n}=-v_{i}^{\max } \psi^{\prime}\left(\xi_{i, j}\right) \Delta x\left(\sum_{k=1}^{+\infty} \omega_{i}^{k} r_{j+k}^{n}-\sum_{k=1}^{+\infty} \omega_{i}^{k} r_{j+k-1}^{n}\right)
$$




$$
\begin{aligned}
& \leq-v_{i}^{\max } \psi^{\prime}\left(\xi_{i, j}\right) \Delta x\left(\sum_{k=1}^{+\infty}\left(\omega_{i}^{k}-\omega_{i}^{k+1}\right) r_{j+k}^{n}-\omega_{i}^{1} r_{j}^{n}\right) \\
& \leq v_{i}^{\max } \Delta x W_{0}\left\|\psi^{\prime}\right\|_{\infty}\left\|r^{n}\right\|_{\infty},
\end{aligned}
$$

for some $\xi_{i, j} \in \mathcal{I}\left(\Delta x \sum_{k=1}^{+\infty} \omega_{i}^{k} r_{j+k-1}^{n}, \Delta x \sum_{k=1}^{+\infty} \omega_{i}^{k} r_{j+k}^{n}\right)$, where we have set $\mathcal{I}(a, b)=$ $[\min \{a, b\}, \max \{a, b\}]$. Therefore $\rho^{n+1,-} \geq 0$ under (2.9).

(ii) Assume $M=1$ and set $V_{j+1 / 2}^{n}:=V_{1, j+1 / 2}^{n}, \omega^{k}:=\omega_{1}^{k}$ and $v^{\max }:=v_{1}^{\max }$. Let us prove the upper bound, the lower one resulting from a symmetric procedure. Define $\bar{\rho}_{j}=\max \left\{\rho_{j}^{n}, \ldots, \rho_{j+N}^{n}\right\}$. Consider first the case $V_{j+1 / 2}^{n} \geq V_{j-1 / 2}^{n}$. Then it is clear that

$$
\rho_{j}^{n+1,-}=\frac{\rho_{j}^{n}}{1+\lambda\left(V_{j+1 / 2}^{n}-V_{j-1 / 2}^{n}\right)} \leq \bar{\rho}_{j}
$$

Consider now the case $V_{j+1 / 2}^{n} \leq V_{j-1 / 2}^{n}$. We note that

$$
\frac{\rho_{j}^{n}}{1+\lambda\left(V_{j+1 / 2}^{n}-V_{j-1 / 2}^{n}\right)} \leq \bar{\rho}_{j} \Longleftrightarrow \bar{\rho}_{j}-\rho_{j}^{n}+\lambda \bar{\rho}_{j}\left(V_{j+1 / 2}^{n}-V_{j-1 / 2}^{n}\right) \geq 0 .
$$

According with $(i)$, we have the estimation

$$
\begin{aligned}
V_{j-1 / 2}^{n}-V_{j+1 / 2}^{n} & =-v^{\max } \psi^{\prime}\left(\xi_{j}\right) \Delta x\left(\sum_{k=1}^{+\infty}\left(\omega^{k}-\omega^{k+1}\right) \rho_{j+k}^{n}-\omega^{1} \rho_{j}^{n}\right) \\
& \leq v^{\max }\left\|\psi^{\prime}\right\|_{\infty} \Delta x\left(\omega^{1} \bar{\rho}_{j}-\omega^{1} \rho_{j}\right) \\
& \leq v^{\max }\left\|\psi^{\prime}\right\|_{\infty} \Delta x\left(\bar{\rho}_{j}-\rho_{j}\right) W_{0} .
\end{aligned}
$$

Finally we obtain that

$$
\bar{\rho}_{j}-\rho_{j}^{n}-\lambda \bar{\rho}_{j}\left(V_{j-1 / 2}^{n}-V_{j+1 / 2}^{n}\right) \geq\left(\bar{\rho}_{j}-\rho_{j}^{n}\right)\left(1-\Delta t v^{\max } \bar{\rho}_{j}\left\|\psi^{\prime}\right\|_{\infty} W_{0}\right) \geq 0
$$

holds if $\Delta t \leq\left(v^{\max }\left\|\psi^{\prime}\right\|_{\infty}\left\|\rho^{n}\right\|_{\infty} W_{0}\right)^{-1}$.

Remark 1. Due to the lack of uniform $\mathbf{L}^{\infty}$ estimates on approximate solutions [10], the time step should in principle be recomputed at each iteration to comply with (2.9). In practice, since the computed solutions stay uniformly bounded in time, it is possible to choose a fixed time step, as we did in Section 4. Moreover, we remark that, in the particular case $M=1$, the maximum principle (2.10) guarantees that $\left\|\rho^{n}\right\|_{\infty} \leq\left\|\rho^{0}\right\|_{\infty}$ for all $n \in \mathbb{N}^{*}$.

\subsection{Remap Step: Antidiffusive scheme}

After the Lagrangian step, the new value $\rho_{i, j}^{n+1,-}$ represents approximate values of the density of the $i$-th class on a moved mesh with new cells $\left[\bar{x}_{j-1 / 2}, \bar{x}_{j+1 / 2}\right]$ for all $j$. To avoid dealing with moving meshes, a so-called remap step is necessary to define the new approximations 
$\rho_{j}^{n+1}$ on the uniform mesh with cells $\left[x_{j-1 / 2}, x_{j+1 / 2}\right]$. This averaging step can equivalently be reformulated by using the solution of the transport equation (2.6) with initial data defined by $\rho_{i, j}^{n+1,-}$ on each cell $\left[x_{j-1 / 2}, x_{j+1 / 2}\right]$, i.e. we consider a numerical scheme in the form

$$
\rho_{i, j}^{n+1}=\rho_{i, j}^{n+1,-}-\bar{V}_{i, j}^{n} \lambda\left(\rho_{i, j+1 / 2}^{n+1,-}-\rho_{i, j-1 / 2}^{n+1,-}\right), \quad i=1, \ldots, M \quad j \in \mathbb{Z} .
$$

Here, $\bar{V}_{i, j}^{n}$ is a velocity value, defined in terms of available density, which will be chosen in such a way that the complete scheme 2.5 plus 2.12 is conservative with respect to (1.1). The quantities $\rho_{i, j+1 / 2}^{n+1,-}$ are numerical fluxes associated with the cell interfaces $x_{j+\frac{1}{2}}$ and will be chosen such that the scheme $(2.12)$ has certain stability and consistency properties. In particular, the choice $\rho_{i, j+1 / 2}^{n+1,-}=\rho_{i, j}^{n+1,-}$ for all $j \in \mathbb{Z}$ produces a diffusive and stable scheme, while $\rho_{i, j+1 / 2}^{n+1,-}=\rho_{i, j+1}^{n+1,-}$ yields an antidiffusive but unstable scheme. For this reason, we proceed as in [4, 12] and we choose $\rho_{i, j+1 / 2}^{n+1,-}$ as close to the anti-diffusive value $\rho_{i, j+1}^{n+1,-}$ as possible, subject to the following consistency condition

$$
m_{i, j+1 / 2}:=\min \left\{\rho_{i, j}^{n+1,-}, \rho_{i, j+1}^{n+1,-}\right\} \leq \rho_{i, j+1 / 2}^{n+1,-} \leq M_{i, j+1 / 2}:=\max \left\{\rho_{i, j}^{n+1,-}, \rho_{i, j+1}^{n+1,-}\right\},
$$

and maximum principle

$$
m_{i, j+1 / 2} \leq \rho_{i, j}^{n+1} \leq M_{i, j+1 / 2},
$$

which resume the properties of the scheme defined by 2.12 .

Let us now define

$$
b_{i, j}^{+}:=M_{i, j+1 / 2}+\frac{\rho_{i, j}^{n+1,-}-M_{i, j+1 / 2}}{\max \left\{v_{i, j-1 / 2}^{n}, v_{i, j+1 / 2}^{n}\right\} \lambda}, \quad B_{i, j}^{+}:=m_{i, j+1 / 2}+\frac{\rho_{i, j}^{n+1,-}-m_{i, j+1 / 2}}{\max \left\{v_{i, j-1 / 2}^{n}, v_{i, j+1 / 2}^{n}\right\} \lambda}
$$

and

$$
a_{i, j+1 / 2}:=\max \left\{b_{i, j}^{+}, m_{i, j+1 / 2}\right\}, \quad A_{i, j+1 / 2}:=\max \left\{B_{i, j}^{+}, M_{i, j+1 / 2}\right\} .
$$

In the next lemma, which is a slight modification of Lemma 4.1 in [5], we summarize the existence and properties of the scheme defined by 2.12 .

Lemma 2. Assume that the following CFL condition holds

$$
\Delta t \leq \frac{\Delta x}{v_{M}^{\max }\|\psi\|_{\infty}}
$$

Then $a_{i, j+1 / 2} \leq \rho_{i, j}^{n+1,-} \leq A_{i, j+1 / 2}$ for all $j \in \mathbb{Z}$, and for any numerical flux that satisfies

$$
\rho_{i, j+1 / 2}^{n+1,-} \in\left[a_{i, j+1 / 2}, A_{i, j+1 / 2}\right], \quad \text { for all } j \in \mathbb{Z},
$$

the scheme 2.12 is $L^{\infty}$-stable, that is

$$
\rho_{i, j}^{n+1} \in \mathcal{I}\left(\rho_{i, j}^{n+1,-}, \rho_{i, j+1}^{n+1,-}\right), \quad \text { for all } j \in \mathbb{Z},
$$

and TVD, i.e.,

$$
\sum_{j \in \mathbb{Z}}\left|\rho_{i, j+1}^{n+1,-}-\rho_{i, j}^{n+1,-}\right| \leq \sum_{j \in \mathbb{Z}}\left|\rho_{i, j+1}^{n}-\rho_{i, j}^{n}\right| .
$$

In particular, for each $j \in \mathbb{Z}$, there exist numbers $\alpha_{i, j} \in[0,1]$ such that

$$
\rho_{i, j}^{n+1,-}=\alpha_{i, j} \rho_{i, j-1 / 2}^{n+1,-}+\left(1-\alpha_{i, j}\right) \rho_{i, j+1 / 2}^{n+1,-}
$$




\subsection{Choice of numerical flux}

In this subsection, we explain how to compute $\rho_{i, j+1 / 2}^{n+1,-}$ for the scalar case $M=1$, the procedure can be applied component-wise in the multi-class case $M>1$. We here proceed as in [5] and consider the so-called U-Bee method proposed in [12] for linear transport equation, which is defined by

$$
\rho_{j+1 / 2}^{n+1,-}:=\rho_{j}^{n+1,-}+\frac{1-\bar{\lambda}_{j}}{2} \varphi_{j}\left(\rho_{j+1}^{n+1,-}-\rho_{j}^{n+1,-}\right),
$$

where $\bar{\lambda}_{j}=\lambda \max \left\{V_{j-1 / 2}^{n}, V_{j+1 / 2}^{n}\right\}, \varphi_{j}:=\varphi\left(R_{j}, \bar{\lambda}_{j}\right)$, with $R_{j}:=\frac{\rho_{j}^{n+1,-}-\rho_{j-1}^{n+1,-}}{\rho_{j+1}^{n+1,-}-\rho_{j}^{n+1,-}}$ and

$$
\varphi(R, \bar{\lambda}):=\varphi^{U B}(R, \bar{\lambda})=\max \left\{0, \min \left\{\frac{2}{1-\lambda}, \frac{2 R}{\lambda}\right\}\right\} .
$$

Similarly, the so-called N-Bee method described, in [4], corresponds to a second-order scheme in space and it is more diffusive that the U-Bee scheme. It is defined as in 2.20 with

$$
\varphi(R, \bar{\lambda}):=\varphi^{N B}(R, \bar{\lambda}):=\max \left\{0, \min \left\{1, \frac{2 R}{\lambda}\right\}, \min \left\{R, \frac{2}{1-\bar{\lambda}}\right\}\right\} .
$$

It is proved in [4] that the numerical flux 2.20 for U-Bee and N-Bee methods satisfies the assumptions of Lemma 2 .

\subsection{Lagrangian-Antidiffusive Remap scheme}

Assume that $\boldsymbol{\rho}^{n}=\left(\rho_{1}^{n}, \ldots, \rho_{M}^{n}\right)$ approximates the solution of (1.1) at time $t=t^{n}$ and we wish to advance this solution to $t^{n+1}=t^{n}+\Delta t$. To this end, two steps are performed successively:

1. Lagrangian step. Consider that $\boldsymbol{\rho}^{n}$ are initial data for $(2.5)$. First, we define the intermediate velocities $V_{i, j+1 / 2}^{n}$ by using (2.3), then we compute the numerical solution $\rho_{i, j}^{n+1,-}$ of equation 2.5) after an evolution over a time interval of length $\Delta t$, using scheme (2.8).

2. Antidiffusive remap step. Solve (2.6) with initial data $\rho_{i, j}^{n+1,-}$ using an antidiffusive scheme 2.12 for a specific choice of $\bar{V}_{i, j}^{n}$, obtaining a numerical solution $\boldsymbol{\rho}^{n+1}$ which approximates the solution of (1.1) a time $t^{n+1}$.

In the next theorem, the choice of $\bar{V}_{i, j}^{n}$ is motivated by the existence of a classical conservative update formula for the whole L-AR scheme (2.5) plus (2.12).

Theorem 1. Under the stability conditions (2.9) and (2.15), there exists a definition of $\bar{V}_{i, j}^{n} \in \mathcal{I}\left(v_{i, j-1 / 2}^{n}, v_{i, j+1 / 2}^{n}\right)$ such that the complete Lagrangian-Antidiffusive remap scheme can be written in the conservative form

$$
\rho_{i, j}^{n+1}=\rho_{i, j}^{n}-\lambda\left(\rho_{i, j+1 / 2}^{n+1,-} V_{i, j+1 / 2}^{n}-\rho_{i, j-1 / 2}^{n+1,-} V_{i, j-1 / 2}^{n}\right), \quad j \in \mathbb{Z} .
$$

Proof. Let $\left\{\rho_{i, j}^{n+1,-}\right\}_{j \in \mathbb{Z}}$, be a solution of 2.5 obtained by scheme (2.8). Using this solution we solve (2.6) by the scheme 2.12), where the value $\bar{V}_{i, j}^{n}$ still needs to be determined in such a way that the resulting scheme is conservative. Replacing $\rho_{i, j}^{n+1,-}$ in 2.12 we obtain

$$
\rho_{i, j}^{n+1}=\rho_{i, j}^{n}-\lambda\left(V_{i, j+1 / 2}^{n}-V_{i, j-1 / 2}^{n}\right) \rho_{i, j}^{n+1,-}-\bar{V}_{i, j}^{n} \lambda\left(\rho_{i, j+1 / 2}^{n+1,-}-\rho_{i, j-1 / 2}^{n+1,-}\right) .
$$


As $\rho_{i, j+1 / 2}^{n+1,-}$ satisfies the assumptions of Lemma 2, there exist $\alpha_{i, j} \in[0,1]$ satisfying 2.19). Setting $\bar{V}_{i, j}^{n}:=\alpha_{i, j} V_{i, j-1 / 2}^{n}+\left(1-\alpha_{i, j}\right) V_{i, j+1 / 2}^{n}$ in (2.24), we obtain 2.23).

Remark 2. Note that, due to 2.13, the numerical flux $F_{i, j+1 / 2}^{n}:=\rho_{i, j+1 / 2}^{n+1,-} V_{i, j+1 / 2}^{n}$ in 2.23) is consistent with the flux $f_{i}(\rho)=\rho_{i} v\left(r * \omega_{i}\right)$.

As a consequence of Lemmas 1 and 2, we have the following property.

Lemma 3. (Positivity) For any $T>0$, under the stability conditions (2.9) and (2.15), the scheme $(2.23)$ is positivity preserving on $[0, T] \times \mathbb{R}$.

Moreover, in the scalar case, we have the following estimates.

Lemma $4\left(\mathbf{L}^{\infty}\right.$ estimate, case $\left.M=1\right)$. Under conditions (2.15) and $(2.9)$, and as a consequence of (2.10) and (2.17), we have

$$
\rho_{j}^{n+1} \in \mathcal{I}\left(\rho_{j-1}^{n}, \rho_{j+1}^{n}\right) \text { for all } j \in \mathbb{Z} .
$$

Lemma 5 (BV estimates, case $M=1)$. Assume (2.15) and

$$
\Delta t \leq \frac{1}{v^{\max }\left\|\psi^{\prime}\right\|_{\infty}\left\|\rho^{0}\right\|_{\infty} W_{0}} .
$$

Let $\boldsymbol{\rho}^{\Delta x}$ be constructed using 2.23. Then for every $T>0$ the following discrete space $B V$ estimate holds

$$
\operatorname{TV}\left(\boldsymbol{\rho}^{\Delta x}(T, \cdot)\right) \leq e^{v^{\max } W_{0}\left\|\rho^{0}\right\|_{\infty}\left(3\left\|\psi^{\prime}\right\|_{\infty}+5\left\|\psi^{\prime \prime}\right\|_{\infty}\left\|\rho^{0}\right\|_{\infty} J_{1}\right) T} \operatorname{TV}\left(\rho^{0}\right) .
$$

Proof. Setting $v(\xi):=v^{\max } \psi(\xi)$, from (2.8) we recover

$$
\rho_{j+1}^{n+1,-}-\rho_{j}^{n+1,-}=\rho_{j+1}^{n}-\rho_{j}^{n}-\lambda \rho_{j+1}^{n+1,-}\left(V_{j+3 / 2}^{n}-V_{j+1 / 2}^{n}\right)+\lambda \rho_{j}^{n+1,-}\left(V_{j+1 / 2}^{n}-V_{j-1 / 2}^{n}\right) .
$$

We have

$$
\begin{aligned}
& -\lambda \rho_{j+1}^{n+1,-}\left(V_{j+3 / 2}^{n}-V_{j+1 / 2}^{n}\right)+\lambda \rho_{j}^{n+1,-}\left(V_{j+1 / 2}^{n}-V_{j-1 / 2}^{n}\right) \\
= & -\lambda \rho_{j+1}^{n+1,-} v^{\prime}\left(\xi_{j+1}\right) \Delta x \sum_{k=1}^{+\infty} \omega^{k}\left(\rho_{j+k+1}^{n}-\rho_{j+k}^{n}\right)+\lambda \rho_{j}^{n+1,-} v^{\prime}\left(\xi_{j}\right) \Delta x \sum_{k=1}^{+\infty} \omega^{k}\left(\rho_{j+k}^{n}-\rho_{j+k-1}^{n}\right) \\
= & -\Delta t\left[\rho_{j+1}^{n+1,-}-\rho_{j}^{n+1,-}\right] v^{\prime}\left(\xi_{j+1}\right) \sum_{k=1}^{+\infty} \omega^{k}\left(\rho_{j+k+1}^{n}-\rho_{j+k}^{n}\right) \\
& -\Delta t \rho_{j}^{n+1,-}\left[v^{\prime}\left(\xi_{j+1}\right)-v^{\prime}\left(\xi_{j}\right)\right] \sum_{k=1}^{+\infty} \omega^{k}\left(\rho_{j+k+1}^{n}-\rho_{j+k}^{n}\right) \\
& -\Delta t \rho_{j}^{n+1,-} v^{\prime}\left(\xi_{j}\right)\left[\sum_{k=1}^{+\infty} \omega^{k}\left(\rho_{j+k+1}^{n}-\rho_{j+k}^{n}\right)-\sum_{k=1}^{+\infty} \omega^{k}\left(\rho_{j+k}^{n}-\rho_{j+k-1}^{n}\right)\right] \\
= & -\Delta t\left[\rho_{j+1}^{n+1,-}-\rho_{j}^{n+1,-}\right] v^{\prime}\left(\xi_{j+1}\right) \sum_{k=1}^{+\infty} \omega^{k}\left(\rho_{j+k+1}^{n}-\rho_{j+k}^{n}\right)
\end{aligned}
$$




$$
\begin{aligned}
& -\Delta t \rho_{j}^{n+1,-}\left[v^{\prime}\left(\xi_{j+1}\right)-v^{\prime}\left(\xi_{j}\right)\right] \sum_{k=1}^{+\infty} \omega^{k}\left(\rho_{j+k+1}^{n}-\rho_{j+k}^{n}\right) \\
& -\Delta t \rho_{j}^{n+1,-} v^{\prime}\left(\xi_{j}\right)\left[\sum_{k=1}^{+\infty} \omega^{k}\left(\rho_{j+k+1}^{n}-\rho_{j+k}^{n}\right)-\sum_{k=1}^{+\infty} \omega^{k}\left(\rho_{j+k}^{n}-\rho_{j+k-1}^{n}\right)\right] .
\end{aligned}
$$

This implies

$$
\begin{aligned}
& {\left[1+\Delta t v^{\prime}\left(\xi_{j+1}\right) \sum_{k=1}^{+\infty} \omega^{k}\left(\rho_{j+k+1}^{n}-\rho_{j+k}^{n}\right)\right]\left(\rho_{j+1}^{n+1,-}-\rho_{j}^{n+1,-}\right) } \\
= & \rho_{j+1}^{n}-\rho_{j}^{n} \\
& -\Delta t \rho_{j}^{n+1,-}\left[v^{\prime}\left(\xi_{j+1}\right)-v^{\prime}\left(\xi_{j}\right)\right] \sum_{k=1}^{+\infty} \omega^{k}\left(\rho_{j+k+1}^{n}-\rho_{j+k}^{n}\right) \\
& -\Delta t \rho_{j}^{n+1,-} v^{\prime}\left(\xi_{j}\right)\left[\sum_{k=1}^{+\infty} \omega^{k}\left(\rho_{j+k+1}^{n}-\rho_{j+k}^{n}\right)-\sum_{k=1}^{+\infty} \omega^{k}\left(\rho_{j+k}^{n}-\rho_{j+k-1}^{n}\right)\right] .
\end{aligned}
$$

Observe that

$$
1+\Delta t v^{\prime}\left(\xi_{j+1}\right) \sum_{k=1}^{+\infty} \omega^{k}\left(\rho_{j+k+1}^{n}-\rho_{j+k}^{n}\right) \geq 1-\Delta t\left\|v^{\prime}\right\|_{\infty}\left\|\rho^{0}\right\|_{\infty} W_{0}
$$

which is positive if $\Delta t \leq\left(\left\|v^{\prime}\right\|_{\infty}\left\|\rho^{0}\right\|_{\infty} W_{0}\right)^{-1}$. Moreover, we have that

$$
v^{\prime}\left(\xi_{j+1}\right)-v^{\prime}\left(\xi_{j}\right)=v^{\prime \prime}\left(\zeta_{j+1 / 2}\right)\left(\xi_{j+1}-\xi_{j}\right),
$$

with $\zeta_{j+1 / 2} \in \mathcal{I}\left(\xi_{j}, \xi_{j+1}\right)$. We can compute

$$
\begin{aligned}
\xi_{j+1}-\xi_{j}= & \vartheta \Delta x \sum_{k=1}^{+\infty} \omega^{k} \rho_{j+k+1}^{n}+(1-\vartheta) \Delta x \sum_{k=1}^{+\infty} \omega^{k} \rho_{j+k}^{n} \\
& -\mu \Delta x \sum_{k=1}^{+\infty} \omega^{k} \rho_{j+k}^{n}-(1-\mu) \Delta x \sum_{k=1}^{+\infty} \omega^{k} \rho_{j+k-1}^{n} \\
= & \vartheta \Delta x \sum_{k=2}^{+\infty} \omega^{k-1} \rho_{j+k}^{n}+(1-\vartheta) \Delta x \sum_{k=1}^{+\infty} \omega^{k} \rho_{j+k}^{n} \\
& -\mu \Delta x \sum_{k=1}^{+\infty} \omega^{k} \rho_{j+k}^{n}+(1-\mu) \Delta x \sum_{k=0}^{+\infty} \omega^{k+1} \rho_{j+k}^{n} \\
= & \Delta x \sum_{k=2}^{+\infty}\left[\vartheta \omega^{k-1}+(1-\vartheta) \omega^{k}-\mu \omega^{k}-(1-\mu) \omega^{k+1}\right] \rho_{j+k}^{n} \\
& +\Delta x\left[(1-\vartheta) \omega^{1} \rho_{j+1}^{n}-\mu \omega^{1} \rho_{j+1}^{n}-(1-\mu) \omega^{1} \rho_{j}^{n}-(1-\mu) \omega^{2} \rho_{j+1}^{n}\right] .
\end{aligned}
$$


By monotonicity of $\omega$ we have

$$
\vartheta \omega^{k-1}+(1-\vartheta) \omega^{k}-\mu \omega^{k}-(1-\mu) \omega^{k+1} \geq 0 .
$$

Taking the absolute values we get

$$
\begin{aligned}
\left|\xi_{j+1}-\xi_{j}\right| & \leq \Delta x\left\|\rho^{0}\right\|_{\infty}\left\{\sum_{k=2}^{+\infty}\left[\vartheta \omega^{k-1}+(1-\vartheta) \omega^{k}-\mu \omega^{k}-(1-\mu) \omega^{k+1}\right]+3 \omega^{1}\right\} \\
& =\Delta x\left\|\rho^{0}\right\|_{\infty}\left\{\vartheta \omega^{1}+(1-\mu) \omega^{2}+3 \omega^{1}\right\} \\
& \leq \Delta x 5\left\|\rho^{0}\right\|_{\infty} W_{0} .
\end{aligned}
$$

Taking the absolute values in (2.27) we get

$$
\begin{aligned}
& \left(1-\Delta t\left\|v^{\prime}\right\|_{\infty}\left\|\rho^{0}\right\|_{\infty} W_{0}\right) \sum_{j}\left|\rho_{j+1}^{n+1,-}-\rho_{j}^{n+1,-}\right| \\
\leq & {\left[1+\left(\left(\sum_{k=1}^{\infty} \rho_{j-k}^{n+1,-}\left|v^{\prime}\left(\xi_{j+1-k}\right)-v^{\prime}\left(\xi_{j-k}\right)\right| \omega^{k}-\rho_{j-k}^{n+1,-} v^{\prime}\left(\xi_{j}\right)\left(\omega^{k}-\omega^{k+1}\right)\right)\right.\right.} \\
\left.\left.-\rho_{j}^{n+1,-} v^{\prime}\left(\xi_{j}\right) \omega^{1}\right) \Delta t\right] \sum_{j}\left|\rho_{j+1}^{n}-\rho_{j}^{n}\right| & {\left[1+5 \Delta t\left\|\rho^{0}\right\|_{\infty}^{2} W_{0}\left\|v^{\prime \prime}\right\| \Delta x \sum_{k=1}^{\infty} \omega^{k}+2 \Delta t W_{0}\left\|\rho^{0}\right\|_{\infty}\left\|v^{\prime}\right\|_{\infty}\right] \sum_{j}\left|\rho_{j+1}^{n}-\rho_{j}^{n}\right| } \\
\leq & {\left[1+\Delta t\left\|\rho^{0}\right\|_{\infty} W_{0}\left(2\left\|v^{\prime}\right\|_{\infty}+5\left\|v^{\prime \prime}\right\|\left\|_{\infty}\right\| \rho^{0} \|_{\infty} J_{1}\right)\right] \sum_{j}\left|\rho_{j+1}^{n}-\rho_{j}^{n}\right|, }
\end{aligned}
$$

which, together with the TVD property of the remap step [4, 5], implies

$$
\begin{aligned}
\operatorname{TV}\left(\rho_{\Delta x}(T, \cdot)\right) & \leq\left(\frac{1+\Delta t\left\|\rho^{0}\right\|_{\infty} W_{0}\left(2\left\|v^{\prime}\right\|_{\infty}+5\left\|v^{\prime \prime}\right\|_{\infty}\left\|\rho^{0}\right\|_{\infty} J_{1}\right)}{1-\Delta t\left\|v^{\prime}\right\|_{\infty}\left\|\rho^{0}\right\|_{\infty} W_{0}}\right)^{\frac{T}{\Delta t}} \operatorname{TV}\left(\rho^{\Delta x}(0, \cdot)\right) \\
& \leq e^{\left\|\rho^{0}\right\|_{\infty} W_{0}\left(3\left\|v^{\prime}\right\|_{\infty}+5\left\|v^{\prime \prime}\right\|_{\infty}\left\|\rho^{0}\right\|_{\infty} J_{1}\right) T} \operatorname{TV}\left(\rho^{0}\right) .
\end{aligned}
$$

Remark 3. Following the ideas of Lemmas 2 and 4 in [10], it is possible to find the local time extension to the above estimates for the case $M>1$.

Next Theorem follows from Theorem 1 and Lemmas 3, 4 and 5 .

Theorem 2 (Convergence to weak solutions, case $M=1$ ). Let us consider the Cauchy problem (1.1)-(1.4) with $M=1, \rho^{0}(x) \in \mathbf{B V}(\mathbb{R} ;[0,1])$, under the assumptions $(\mathbf{H 1})$ - (H3). If (2.15) and 2.9) hold, then the approximate solution $\rho^{\Delta x}$ constructed by the scheme (2.23) converges to a weak solution of (1.1) - (1.4). 
Proof. Under conditions (2.15) and (2.9), the approximate solutions $\rho^{\Delta x}$ constructed by the numerical scheme (2.23) are uniformly bounded and uniformly bounded total variation. The result follows by standard application of Helly's Theorem, see [16, Proposition 4.1] or [13, Lemma 5.6].

\section{Two simple schemes for the non-local multi-class traffic flow model}

In Section 4, we consider the following conservative schemes for the multi-class model (1.1) in the form

$$
\rho_{i, j}^{n+1}=\rho_{i, j}^{n}-\lambda\left(F_{i, j+1 / 2}^{n}-F_{i, j-1 / 2}^{n}\right), \quad i=1, \ldots, M .
$$

First, we consider the Godunov-type scheme, which was introduced in [14] in the scalar case and then extended to (1.1) in [10], with numerical flux

$$
F_{i, j+1 / 2}^{n}:=\rho_{i, j}^{n} V_{i, j+1 / 2}^{n} .
$$

We recall that for scheme (3.1)-(3.2) the positivity is guaranteed if

$$
\lambda \leq \frac{1}{v_{M}^{\max }\|\psi\|_{\infty}} .
$$

We consider also the approximate solutions constructed via the following adapted Lax-Friedrichs flux, that was used [3, 9] in the scalar case and in [10] for system (1.1):

$$
F_{i, j+1 / 2}^{n}:=\frac{1}{2}\left(\rho_{i, j}^{n} V_{i, j-1 / 2}^{n}+\rho_{i, j+1}^{n} V_{i, j-3 / 2}^{n}\right)+\frac{\alpha}{2}\left(\rho_{i, j}^{n}-\rho_{i, j+1}^{n}\right),
$$

where $\alpha \geq v_{M}^{\max }\|\psi\|_{\infty}$ is the viscosity coefficient and $\lambda \alpha \leq 1$ the CFL condition.

\subsection{A second-order Godunov scheme}

Schemes (3.1)-(3.2) and (3.1)-(3.3) are only first-order accurate. We propose here a secondorder accuracy scheme, constructed using MUSCL-type variable extrapolation and RungeKutta temporal differencing. To implement it, we approximate $\rho_{i}\left(x, t^{n}\right)$ by a piecewise linear functions in each cell, i.e. $\hat{\rho}_{i, j}\left(x, t^{n}\right)=\rho_{i, j}^{n}+\sigma_{i, j}^{n}\left(x-x_{j}\right)$, where the slopes $\sigma_{i, j}^{n}$ are calculated via the generalized minmod limiter, i.e.

$$
\sigma_{i, j}^{n}=\frac{1}{\Delta x} \operatorname{minmod}\left(\vartheta\left(\rho_{i, j}^{n}-\rho_{i, j-1}^{n}\right), \frac{1}{2}\left(\rho_{i, j+1}^{n}-\rho_{i, j-1}^{n}\right), \vartheta\left(\rho_{i, j+1}^{n}-\rho_{i, j}^{n}\right)\right),
$$

where $\vartheta \in[1,2]$ and

$$
\operatorname{minmod}(a, b, c):= \begin{cases}\operatorname{sgn}(a) \min \{|a|,|b|,|c|\} & \text { if } \operatorname{sgn}(c)=\operatorname{sgn}(b)=\operatorname{sgn}(a) \\ 0 & \text { otherwise. }\end{cases}
$$

This extrapolation enables one to define left and right values at the cell interfaces respectively by

$$
\rho_{i, j+1 / 2,}^{L}:=\hat{\rho}_{i, j}\left(x_{j}+\Delta x / 2, t^{n}\right)=\rho_{i, j}^{n}+\sigma_{i, j}^{n} \Delta x / 2,
$$




$$
\rho_{i, j-1 / 2,}^{R}:=\hat{\rho}_{i, j}\left(x_{j}-\Delta x / 2, t^{n}\right)=\rho_{i, j}^{n}-\sigma_{i, j}^{n} \Delta x / 2 .
$$

In order to define the corresponding velocity approximations, we set

$$
\hat{r}_{j+k}^{n}=\sum_{i=1}^{M} \hat{\rho}_{i, j+k}^{n}=r_{j+k}^{n}+\Theta_{j+k}^{n}\left(x-x_{j+k}\right),
$$

where $\Theta_{j+k}^{n}:=\sum_{i=1}^{M} \sigma_{i, j+k}^{n}$, and

$$
\hat{V}_{i, j+1 / 2}^{n}:=v_{i}\left(\left(\hat{r} * w_{i}\right)\left(t^{n}, x_{j+1 / 2}\right)\right)=v_{i}^{\max } \psi\left(\Delta x \sum_{k=1}^{+\infty} \omega_{i}^{k} r_{j+k}^{n}+\Delta x \sum_{k=1}^{+\infty} \tilde{\omega}_{i, j}^{k} \Theta_{j+k}^{n}\right)
$$

for $i=1, \ldots, M, j \in \mathbb{Z}$, where $\tilde{\omega}_{i, j}^{k}:=\frac{1}{\Delta x} \int_{-\Delta x / 2}^{\Delta x / 2} y \omega_{i}(y+(k-1 / 2) \Delta x) \mathrm{d} y$. The MUSCL version of the $i$-th flux component thus reads

$$
f_{i, j+1 / 2}^{n}:=\rho_{i, j+1 / 2}^{L} \hat{V}_{i, j+1 / 2}^{n} .
$$

To achieve formal second-order accuracy also in time, we use second-order RungeKutta (RK) time stepping. More precisely, if we write our scheme with first-order Euler time differences and second-order spatial differences formally as

$$
\boldsymbol{\rho}_{j}^{n+1}=\boldsymbol{\rho}_{j}^{n}-\lambda L_{j}\left(\boldsymbol{\rho}^{n}\right):=\boldsymbol{\rho}_{j}^{n}-\lambda\left(\mathbf{F}_{j+1 / 2}^{n}-\mathbf{F}_{j-1 / 2}^{n}\right),
$$

then the RK version takes the following two-step form

$$
\left\{\begin{array}{l}
\boldsymbol{\rho}_{j}^{(1)}=\boldsymbol{\rho}_{j}^{n}-\lambda L_{j}\left(\boldsymbol{\rho}^{n}\right) \\
\boldsymbol{\rho}_{j}^{n+1}=\frac{1}{2}\left(\boldsymbol{\rho}_{j}^{n}+\boldsymbol{\rho}_{j}^{(1)}\right)-\frac{\lambda}{2} L_{j}\left(\boldsymbol{\rho}_{j}^{(1)}\right) .
\end{array}\right.
$$

Lemma 6. For any $T>0$, under the CFL condition

$$
\Delta t \leq \frac{\Delta x}{2 v_{M}^{\max }\|\psi\|_{\infty}}
$$

the scheme 3.6 is positivity preserving on $\mathbb{R} \times[0, T]$.

Proof. Let us assume that $\rho_{i, j}^{n} \geq 0$ for $j \in \mathbb{Z}$ and $i=1, \ldots, M$. The positivity of the reconstructed values $\rho_{i, j+1 / 2}^{L}$ and $\rho_{i, j+1 / 2}^{L}$ is guaranteed by the positivity preserving property of the chosen limiter [19, 20]. It suffices to prove that $\rho_{i, j}^{n+1} \geq 0$ in $(3.5)$. Due to $\rho_{i, j}^{n}=$ $\frac{1}{2}\left(\rho_{i, j-1 / 2}^{R}+\rho_{i, j+1 / 2}^{L}\right)$, the $i$-th term in 3.5 can be written in the form

$$
\rho_{i, j}^{n+1}=\frac{1}{2} \rho_{i, j-1 / 2}^{R}+\left(\frac{1}{2}-\lambda \hat{V}_{i, j+1 / 2}^{n}\right) \rho_{i, j+1 / 2}^{L}+\lambda \rho_{i, j-1 / 2}^{L} \hat{V}_{i, j-1 / 2}^{n} \geq 0,
$$

under the CFL condition (3.7). 


\section{Numerical results}

In this section, we solve (1.1) numerically in the intervals $x \in[a, b]$ with suitable boundary conditions and $t \in[0, T]$, for values of $a, b$ and $T$ specified later. We propose several test cases to illustrate the behaviour of the L-AR schemes (2.20) and (2.21) in comparison with first-order Lax-Friedrichs (Lax-F) (3.1)- $(3.3)$ and Godunov (3.1)-(3.2) schemes and the second-order Godunov scheme (Godunov2) (3.6). As benchmarks, we consider the numerical examples studied in [14, Section 4.2] for the scalar case and in [10, Sections 4.1-4.2] for the multi-class system case.

From now on, we refer to the numerical scheme (2.23) applied in a component-wise manner with velocities 2.3 and $\rho_{i, j+1 / 2}^{n+1,-}$ computed with (2.20) and 2.21) (respectively (2.22) as LUBee (respectively L-NBee) scheme. For each integration, we set $\Delta t$ to satisfy the most restrictive CFL condition (3.7). Clearly, the computational bottleneck in these schemes is the discrete convolution term (2.3) corresponding to 1.3 . This is a classical problem in scientific computing, which can be effectively evaluated using fast convolution algorithms, mainly based on Fast Fourier Transforms [21].

For each numerical test, we specify the type of boundary conditions that are imposed. Given a uniform partition of $[a, b],\left\{I_{j}\right\}_{j=1}^{N}$ with $\Delta x=(b-a) / N$, for absorbing boundary conditions, we define $\rho_{i, j}^{n}$ in the ghost cells for $i=1, \ldots, M$ as follows:

$$
\rho_{i, 0}^{n}=\rho_{i, 1}^{n}, \quad \text { and } \quad \rho_{i, N+j}^{n}=\rho_{i, N}^{n}, \quad \text { for } j=1,2, \ldots, L,
$$

and for periodic boundary conditions,

$$
\rho_{i, 0}^{n}=\rho_{i, N}^{n}, \quad \text { and } \quad \rho_{i, N+j}^{n}=\rho_{i, j}^{n}, \quad \text { for } j=1,2, \ldots, L,
$$

with $L>\max _{i=1, \ldots, M} \eta_{i} / \Delta x$.

Since we cannot compute the exact solution explicitly, we use the second-order Godunov scheme with a refined mesh to obtain a reference solution. The $\mathbf{L}^{\mathbf{1}}$-error for the cell average is given by

$$
L^{1}(\Delta x)=\sum_{i=1}^{M}\left(\frac{1}{N} \sum_{j=1}^{N}\left|\bar{\rho}_{i, j}-\bar{\rho}_{i, j}^{r e f}\right|\right),
$$

where $\bar{\rho}_{i, j}$ and $\bar{\rho}_{i, j}^{r e f}$ are the cell averages of the numerical approximation and the reference solution respectively. The Experimental Order of Accuracy (E.O.A.) is naturally defined by

$$
\gamma(\Delta x)=\log _{2}\left(L^{1}(\Delta x) / L^{1}(\Delta x / 2)\right) .
$$

\subsection{Test 1, scalar case}

We consider the problem (1.1) for $M=1$, with initial datum

$$
\rho_{0}(x)= \begin{cases}1, & \text { if } 1 / 3 \leq x \leq 2 / 3 \\ \frac{1}{3}, & \text { otherwise, }\end{cases}
$$

for $x \in[0,1]$, with absorbing boundary conditions, and different non-increasing kernel functions with $\eta=0.1$. In Figure 1, we display the numerical approximations obtained with the schemes presented in the previous sections, computed with $1 / \Delta x=80$ at $T=0.1$. 


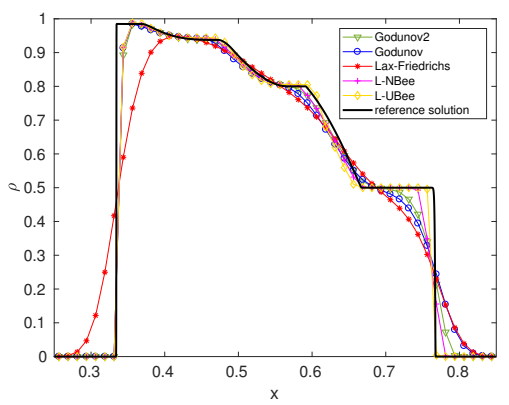

(a)

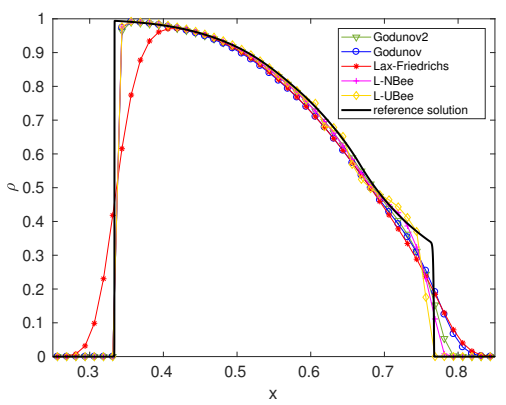

(b)

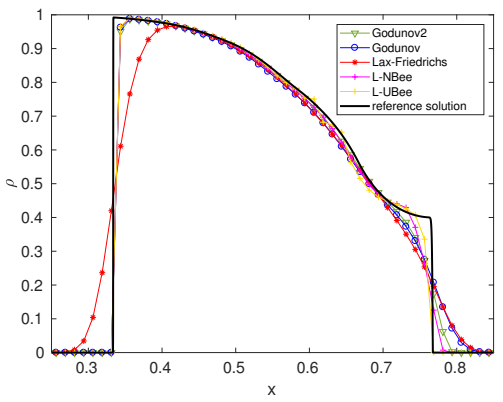

(c)

Figure 1: Test 1: Comparison of the numerical solutions at $T=0.1$ corresponding to the initial condition (4.1), computed with $1 / \Delta x=80$ and different kernel functions. (a) $\omega(x)=1 / \eta$, (b) $\omega(x)=2(\eta-x) / \eta^{2}$, (c) $\omega(x)=3\left(\eta^{2}-x^{2}\right) /\left(2 \eta^{3}\right)$.

Fig. 1a show the result for $\omega(x)=1 / \eta$, Fig. $1 \mathrm{~b}$ for $\omega(x)=2(\eta-x) / \eta^{2}$ and Fig. $1 \mathrm{c}$ for $\omega(x)=3\left(\eta^{2}-x^{2}\right) /\left(2 \eta^{3}\right)$. The reference solution is computed with $1 / \Delta x=10240$. The numerical solutions obtained with L-UBee and L-NBee approximate adequately shocks and rarefaction waves according to the theoretical results of Theorem 2, In particular, concerning the shock waves, L-AR schemes capture the reference solution better than the second-order Godunov scheme, whereas the solutions computed with Lax-Friedrichs and Godunov schemes are more diffusive. In the presence of rarefaction waves, L-UBee scheme produces "staircaising" due to the particular choice of the antidiffusive scheme. We can observe the same "staircaising" phenomena also for the linear advection and other equations [4, 5].

Table 1 shows the approximate $L^{1}(\Delta x)$-errors and the numerical orders of accuracy $\gamma(\Delta x)$ for the different schemes. We computed numerical approximations with $1 / \Delta x=40 \times 2^{q}$ for $q=1,2, \ldots, 5$. Clearly, the error of the L-AR schemes decreases when the mesh is refined and we observe that for each level of refinement, the $\mathbf{L}^{\mathbf{1}}$-error of the L-AR schemes is smaller than the respective errors of Lax-Friedrichs and Godunov schemes. In conclusion, when the solution presents discontinuities, we can compare the performances of the L-AR schemes with those of a second-order scheme.

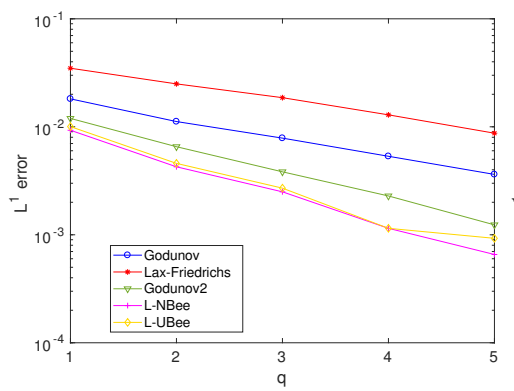

(a)

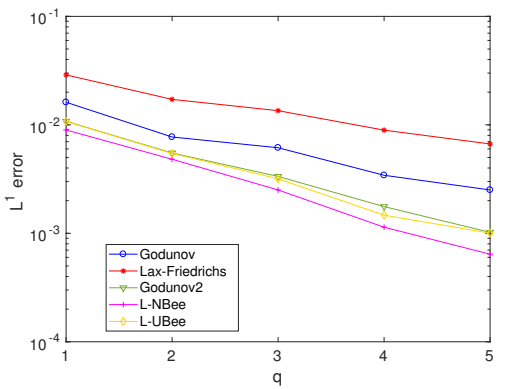

(b)

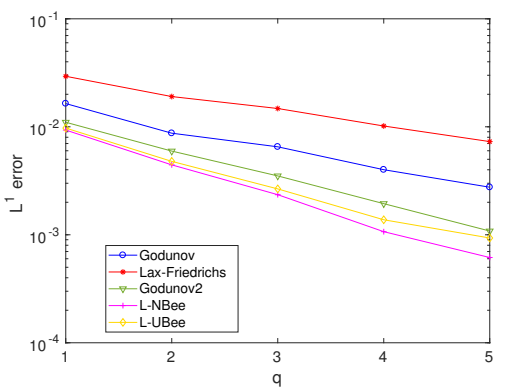

(c)

Figure 2: Test 1. Initial condition (4.1). Approximate $\mathbf{L}^{\mathbf{1}}$-error for different numerical schemes with: (a) constant kernel function $\omega(x)=1 / \eta$, (b) decreasing kernel function $\omega(x)=2(\eta-$ $x) / \eta^{2}$, (c) concave kernel function $\omega(x)=3\left(\eta^{2}-x^{2}\right) /\left(2 \eta^{3}\right)$.

Now, in order to determine the correct order of accuracy of the L-AR schemes, we consider 


\begin{tabular}{|c|c|c|c|c|c|c|c|}
\hline Scheme & & \multicolumn{2}{|c|}{$\omega(x)=1 / \eta$} & \multicolumn{2}{c|}{$\omega(x)=2(\eta-x) / \eta^{2}$} & \multicolumn{2}{c|}{$\omega(x)=3\left(\eta^{2}-x^{2}\right) /\left(2 \eta^{3}\right)$} \\
\hline & $1 / \Delta x$ & $\mathbf{L}^{1}-$ error & $\gamma(\Delta x)$ & $\mathbf{L}^{1}-$ error & $\gamma(\Delta x)$ & $\mathbf{L}^{1}-$ error & $\gamma(\Delta x)$ \\
\hline Godunov & 80 & $1.81 \mathrm{e}-02$ & - & $1.62 \mathrm{e}-02$ & - & $1.64 \mathrm{e}-02$ & - \\
& 160 & $1.12 \mathrm{e}-02$ & $6.98 \mathrm{e}-01$ & $7.73 \mathrm{e}-0.3$ & 1.06 & $8.72 \mathrm{e}-03$ & $9.11 \mathrm{e}-01$ \\
& 320 & $7.85 \mathrm{e}-03$ & $5.10 \mathrm{e}-01$ & $6.15 \mathrm{e}-03$ & $3.29 \mathrm{e}-01$ & $6.53 \mathrm{e}-03$ & $4.19 \mathrm{e}-01$ \\
& 640 & $5.33 \mathrm{e}-03$ & $5.58 \mathrm{e}-01$ & $3.43 \mathrm{e}-03$ & $8.43 \mathrm{e}-01$ & $4.01 \mathrm{e}-03$ & $7.04 \mathrm{e}-01$ \\
& 1280 & $3.62 \mathrm{e}-03$ & $5.58 \mathrm{e}-01$ & $2.51 \mathrm{e}-0.3$ & $4.50 \mathrm{e}-01$ & $2.76 \mathrm{e}-03$ & $5.39 \mathrm{e}-01$ \\
\hline Lax-F & 80 & $3.48 \mathrm{e}-02$ & - & $2.89 \mathrm{e}-02$ & - & $2.94 \mathrm{e}-02$ & - \\
& 160 & $2.50 \mathrm{e}-02$ & $4.81 \mathrm{e}-01$ & $1.72 \mathrm{e}-02$ & $7.50 \mathrm{e}-01$ & $1.91 \mathrm{e}-02$ & $6.23 \mathrm{e}-01$ \\
& 320 & $1.86 \mathrm{e}-02$ & $4.24 \mathrm{e}-01$ & $1.35 \mathrm{e}-02$ & $3.46 \mathrm{e}-01$ & $1.48 \mathrm{e}-02$ & $3.67 \mathrm{e}-01$ \\
& 640 & $1.29 \mathrm{e}-02$ & $5.28 \mathrm{e}-01$ & $8.94 \mathrm{e}-03$ & $5.7 \mathrm{e}-01$ & $1.02 \mathrm{e}-02$ & $5.38 \mathrm{e}-01$ \\
& 1280 & $8.72 \mathrm{e}-03$ & $5.64 \mathrm{e}-01$ & $6.670 \mathrm{e}-03$ & $4.23 \mathrm{e}-01$ & $7.30 \mathrm{e}-03$ & $4.70 \mathrm{e}-01$ \\
\hline L-NBee & 80 & $9.30 \mathrm{e}-03$ & - & $8.93 \mathrm{e}-03$ & - & $9.24 \mathrm{e}-03$ & - \\
& 160 & $4.29 \mathrm{e}-03$ & 1.11 & $4.78 \mathrm{e}-03$ & $9.01 \mathrm{e}-01$ & $4.50 \mathrm{e}-03$ & $1.03 \mathrm{e}-01$ \\
& 320 & $2.51 \mathrm{e}-03$ & $7.47 \mathrm{e}-01$ & $2.52 \mathrm{e}-03$ & $9.27 \mathrm{e}-01$ & $2.37 \mathrm{e}-03$ & $9.25 \mathrm{e}-01$ \\
& 640 & $1.58 \mathrm{e}-03$ & 1.11 & $1.15 \mathrm{e}-03$ & 1.13 & $1.08 \mathrm{e}-03$ & 1.13 \\
& 1280 & $6.57 \mathrm{e}-04$ & $8.17 \mathrm{e}-01$ & $6.46 \mathrm{e}-04$ & $8.31 \mathrm{e}-01$ & $6.19 \mathrm{e}-04$ & $8.05 \mathrm{e}-01$ \\
\hline L-UBee & 80 & $1.00 \mathrm{e}-02$ & - & $8.90 \mathrm{e}-03$ & - & $9.09 \mathrm{e}-03$ & - \\
& 160 & $4.58 \mathrm{e}-03$ & 1.13 & $4.40 \mathrm{e}-03$ & 1.02 & $4.82 \mathrm{e}-03$ & $9.16 \mathrm{e}-01$ \\
& 320 & $2.7 \mathrm{e}-03$ & $7.62 \mathrm{e}-01$ & $2.87 \mathrm{e}-03$ & $6.61 \mathrm{e}-01$ & $2.62 \mathrm{e}-03$ & $8.80 \mathrm{e}-01$ \\
& 640 & $1.15 \mathrm{e}-03$ & 1.23 & $1.38 \mathrm{e}-03$ & 1.05 & $1.37 \mathrm{e}-03$ & $9.30 \mathrm{e}-01$ \\
& 1280 & $9.48 \mathrm{e}-04$ & $2.76 \mathrm{e}-01$ & $9.69 \mathrm{e}-04$ & $5.13 \mathrm{e}-01$ & $9.00 \mathrm{e}-04$ & $6.11 \mathrm{e}-01$ \\
\hline Godunov2 & 80 & $1.20 \mathrm{e}-02$ & - & $1.08 \mathrm{e}-02$ & - & $1.01 \mathrm{e}-02$ & - \\
& 160 & $6.54 \mathrm{e}-03$ & $8.70 \mathrm{e}-01$ & $5.5 \mathrm{e}-03$ & $9.71 \mathrm{e}-01$ & $5.96 \mathrm{e}-03$ & $8.86 \mathrm{e}-01$ \\
& 320 & $3.82 \mathrm{e}-03$ & $7.73 \mathrm{e}-01$ & $3.35 \mathrm{e}-03$ & $7.17 \mathrm{e}-01$ & $3.51 \mathrm{e}-03$ & $7.64 \mathrm{e}-01$ \\
& 640 & $2.29 \mathrm{e}-03$ & $7.42 \mathrm{e}-01$ & $1.76 \mathrm{e}-03$ & $9.25 \mathrm{e}-01$ & $1.94 \mathrm{e}-03$ & $8.53 \mathrm{e}-01$ \\
& 1280 & $1.23 \mathrm{e}-03$ & $8.89 \mathrm{e}-01$ & $1.02 \mathrm{e}-03$ & $7.87 \mathrm{e}-01$ & $1.08 \mathrm{e}-03$ & $8.42 \mathrm{e}-01$ \\
\hline
\end{tabular}

Table 1: Test 1. Approximate $\mathbf{L}^{\mathbf{1}}$-error and E.O.A. $\gamma$ for different numerical schemes and with different kernel functions and $\eta=0.1$ corresponding to the initial condition (4.1).

a smooth initial datum

$$
\rho_{0}(x)=0.5+0.4 \sin (\pi x)
$$

for $x \in[-1,1]$, with periodic boundary conditions and compute the numerical approximation at $T=0.15$ for different kernel functions with $\eta=0.1$. The reference solution is computed with $1 / \Delta x=10240$. In Table 2 and Figure 3 we compute the $L^{1}$-error and E.O.A. $\gamma(\Delta x)$. We recover the correct order of accuracy for the second-order Godunov scheme. Instead, we obtain just first-order accuracy for L-AR schemes. However, it is worth underlying that the $\mathbf{L}^{\mathbf{1}}$-error of the L-NBee scheme is smaller than the corresponding error for Lax-Friedrichs and Godunov schemes. For the L-UBee scheme, we obtain first order accuracy and the $L^{1}$-error for each level of refinement is bigger than the error of the other first order numerical schemes, due to the antidiffusive property of the UBee scheme.

\subsection{Test 2. Cars and trucks mixed traffic}

In this test case, we consider a stretch of road populated by cars and trucks as in the example proposed in [10, Section 4.1]. The space domain is given by the interval $[-1,1]$ and we impose absorbing conditions at the boundaries. The dynamics is described by the equation (1.1) with 


\begin{tabular}{|c|c|c|c|c|c|c|c|}
\hline Scheme & & \multicolumn{2}{|c|}{$\omega(x)=1 / \eta$} & \multicolumn{2}{c|}{$\omega(x)=2(\eta-x) / \eta^{2}$} & \multicolumn{2}{c|}{$\omega(x)=3\left(\eta^{2}-x^{2}\right) /\left(2 \eta^{3}\right)$} \\
\hline & $1 / \Delta x$ & $\mathbf{L}^{1}-$ error & $\gamma(\Delta x)$ & $\mathbf{L}^{1}-$ error & $\gamma(\Delta x)$ & $\mathbf{L}^{1}-$ error & $\gamma(\Delta x)$ \\
\hline Godunov & 80 & $1.28 \mathrm{e}-03$ & - & $1.33 \mathrm{e}-03$ & - & $1.33 \mathrm{e}-03$ & - \\
& 160 & $6.44 \mathrm{e}-04$ & $9.88 \mathrm{e}-01$ & $6.73 \mathrm{e}-0.4$ & $9.95 \mathrm{e}-01$ & $6.68 \mathrm{e}-04$ & $9.94 \mathrm{e}-01$ \\
& 320 & $3.23 \mathrm{e}-04$ & $9.94 \mathrm{e}-01$ & $3.38 \mathrm{e}-04$ & $9.97 \mathrm{e}-01$ & $3.34 \mathrm{e}-04$ & $9.97 \mathrm{e}-01$ \\
& 640 & $1.62 \mathrm{e}-04$ & $9.97 \mathrm{e}-01$ & $1.69 \mathrm{e}-04$ & $9.99 \mathrm{e}-01$ & $1.67 \mathrm{e}-04$ & $9.98 \mathrm{e}-01$ \\
& 1280 & $8.11 \mathrm{e}-05$ & $9.98 \mathrm{e}-01$ & $8.47 \mathrm{e}-0.5$ & $9.99 \mathrm{e}-01$ & $8.38 \mathrm{e}-05$ & $9.99 \mathrm{e}-01$ \\
\hline Lax-F & 80 & $1.58 \mathrm{e}-03$ & - & $1.92 \mathrm{e}-03$ & - & $1.76 \mathrm{e}-03$ & - \\
& 160 & $7.24 \mathrm{e}-04$ & 1.12 & $8.14 \mathrm{e}-04$ & 1.24 & $7.73 \mathrm{e}-04$ & 1.18 \\
& 320 & $3.46 \mathrm{e}-04$ & 1.07 & $3.70 \mathrm{e}-04$ & 1.14 & $3.59 \mathrm{e}-04$ & 1.10 \\
& 640 & $1.69 \mathrm{e}-04$ & 1.03 & $1.77 \mathrm{e}-04$ & 1.06 & $1.74 \mathrm{e}-04$ & 1.05 \\
& 1280 & $8.35 \mathrm{e}-05$ & 1.02 & $8.67 \mathrm{e}-04$ & 1.03 & $8.55 \mathrm{e}-05$ & 1.02 \\
\hline L-NBee & 80 & $4.55 \mathrm{e}-04$ & - & $4.30 \mathrm{e}-04$ & - & $4.36 \mathrm{e}-04$ & - \\
& 160 & $2.23 \mathrm{e}-04$ & 1.02 & $2.24 \mathrm{e}-04$ & $9.43 \mathrm{e}-01$ & $2.24 \mathrm{e}-04$ & $9.65 \mathrm{e}-01$ \\
& 320 & $1.10 \mathrm{e}-04$ & 1.01 & $1.14 \mathrm{e}-04$ & $9.72 \mathrm{e}-01$ & $1.13 \mathrm{e}-04$ & $9.83 \mathrm{e}-01$ \\
& 640 & $5.49 \mathrm{e}-04$ & 1.01 & $5.76 \mathrm{e}-05$ & $9.86 \mathrm{e}-01$ & $5.69 \mathrm{e}-05$ & $9.92 \mathrm{e}-01$ \\
& 1280 & $2.74 \mathrm{e}-05$ & 1.00 & $2.89 \mathrm{e}-05$ & $9.93 \mathrm{e}-01$ & $2.85 \mathrm{e}-05$ & $9.96 \mathrm{e}-01$ \\
\hline L-UBee & 80 & $2.30 \mathrm{e}-03$ & - & $2.14 \mathrm{e}-03$ & - & $2.16 \mathrm{e}-03$ & - \\
& 160 & $1.75 \mathrm{e}-03$ & $3.96 \mathrm{e}-01$ & $1.23 \mathrm{e}-03$ & $7.97 \mathrm{e}-01$ & $1.26 \mathrm{e}-03$ & $7.72 \mathrm{e}-01$ \\
& 320 & $1.48 \mathrm{e}-03$ & $2.49 \mathrm{e}-01$ & $1.18 \mathrm{e}-03$ & $5.59 \mathrm{e}-02$ & $1.20 \mathrm{e}-03$ & $8.05 \mathrm{e}-02$ \\
& 640 & $9.82 \mathrm{e}-04$ & $5.89 \mathrm{e}-01$ & $8.39 \mathrm{e}-04$ & $4.98 \mathrm{e}-01$ & $8.41 \mathrm{e}-04$ & $5.09 \mathrm{e}-01$ \\
& 1280 & $5.06 \mathrm{e}-04$ & $9.56 \mathrm{e}-01$ & $4.53 \mathrm{e}-04$ & $8.88 \mathrm{e}-01$ & $4.63 \mathrm{e}-04$ & $8.61 \mathrm{e}-01$ \\
\hline Godunov2 & 80 & $2.86 \mathrm{e}-05$ & - & $2.89 \mathrm{e}-05$ & - & $2.89 \mathrm{e}-05$ & - \\
& 160 & $6.80 \mathrm{e}-06$ & 2.07 & $6.74 \mathrm{e}-06$ & 2.10 & $6.76 \mathrm{e}-06$ & 2.09 \\
& 320 & $1.53 \mathrm{e}-06$ & 2.15 & $1.53 \mathrm{e}-06$ & 2.14 & $1.53 \mathrm{e}-06$ & 2.14 \\
& 640 & $3.42 \mathrm{e}-07$ & 2.16 & $3.42 \mathrm{e}-07$ & 2.16 & $3.41 \mathrm{e}-07$ & 2.16 \\
& 1280 & $7.72 \mathrm{e}-08$ & 2.15 & $7.75 \mathrm{e}-08$ & 2.14 & $7.73 \mathrm{e}-08$ & 2.14 \\
\hline
\end{tabular}

Table 2: Test 1. Approximate $\mathbf{L}^{\mathbf{1}}$-error and E.O.A. $\gamma$, with smooth initial condition $(4.2)$ and different kernels function with $\eta=0.1$.

$M=2$, and the following initial conditions and parameter values

$$
\begin{array}{llll}
\rho_{1}(0, x)=0.5 \chi_{[-0.6,-0.1]}(x), & \omega_{1}(x)=\frac{2}{\eta_{1}}\left(1-\frac{x}{\eta_{1}}\right), & \eta_{1}=0.3, & v_{1}^{\max }=0.8, \\
\rho_{2}(0, x)=0.5 \chi_{[-0.9,-0.6]}(x), & \omega_{2}(x)=\frac{2}{\eta_{2}}\left(1-\frac{x}{\eta_{2}}\right), & \eta_{2}=0.1, & v_{2}^{\max }=1.3 .
\end{array}
$$

In this setting, $\rho_{1}(t, x)$ and $\rho_{2}(x, t)$ describe the density of trucks and cars respectively. We have a red traffic light located at $x=-0.1$, which turns green at the initial time $t=0$. In Figure 4, we display the reference solution of equation (1.1) with initial conditions and parameters 4.3$)$, computed with with $1 / \Delta x=5120$ at increasing time instants $(T=0.25$ in Fig. $4 \mathrm{a}, T=0.5$ in Fig. $4 \mathrm{~b}$ and $T=1$ in Fig. $4 \mathrm{c}$. We can observe that cars, initially located behind trucks, overtake the slower class while impacting its flow.

In Figure 5, we display separately the two density components of the approximate solutions computed using all the considered schemes with $1 / \Delta x=80$, compared to the reference solution of Figure 4. The numerical tests indicate that for $M>1$, the L-AR solutions are anti-diffusive for each class and they keep this anti-diffusive behavior for the whole simulation time. We observe that the L-NBee solution approaches very well the reference solution for each class at different times. On the other hand, the L-UBee solution shows "stairs" in the presence of rarefaction-waves.

In Table 3 and Figure 6, we compute the approximate $\mathbf{L}^{\mathbf{1}}$-error and the E.O.A at time 


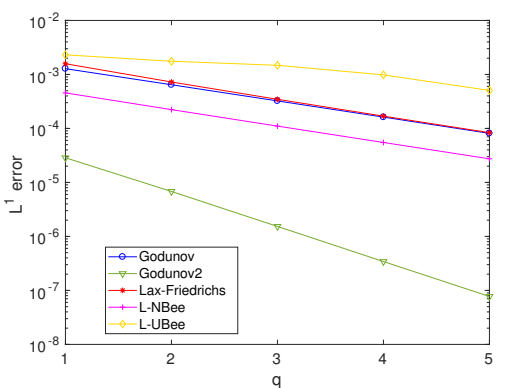

(a)

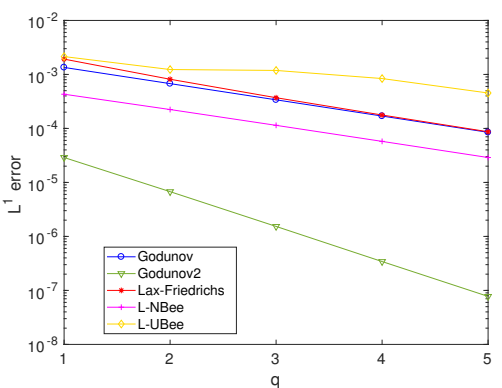

(b)

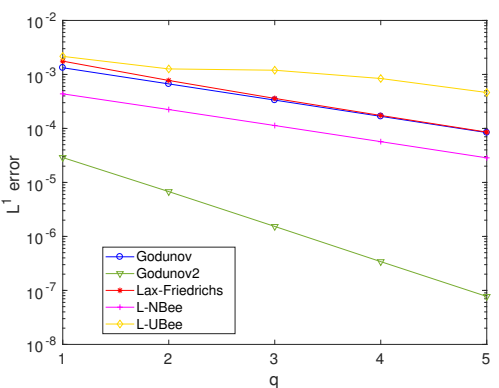

(c)

Figure 3: Test 1. Initial condition (4.2). Approximate $\mathbf{L}^{\mathbf{1}}$-error for different numerical schemes with: (a) constant kernel function $\omega(x)=1 / \eta$, (b) decreasing kernel function $\omega(x)=2(\eta-$ $x) / \eta^{2}$, concave kernel function $\omega(x)=3\left(\eta^{2}-x^{2}\right) /\left(2 \eta^{3}\right)(\mathrm{c})$.

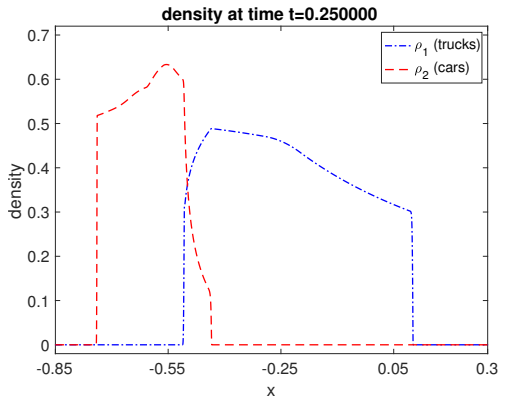

(a)

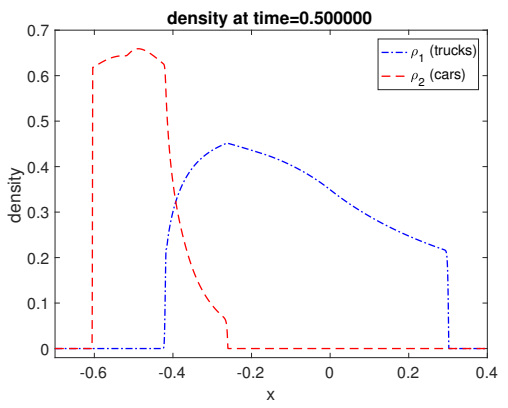

(b)

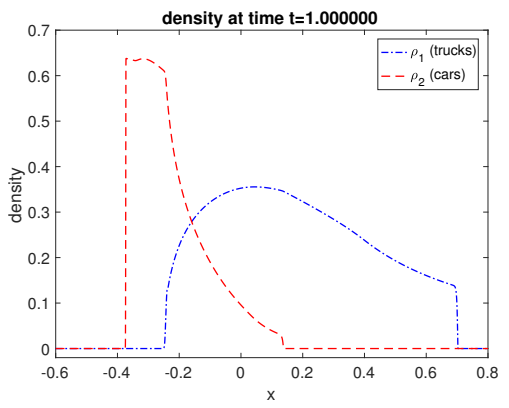

(c)

Figure 4: Test 2: Density profiles corresponding to (1.1)-(4.3), computed by second-order Godunov scheme with $1 / \Delta x=5120$, at different times.

$T=0.5$. We observe that the performance of L-AR schemes are comparable with those of the second-order Godunov scheme. In particular, we have that the L-NBee $\mathbf{L}^{\mathbf{1}}$-error is the smallest for each level of refinement.

\begin{tabular}{|c|c|c|c|c|c|c|c|c|c|c|}
\hline $1 / \Delta x$ & \multicolumn{2}{|c|}{ Godunov } & \multicolumn{2}{c|}{ Lax-F } & \multicolumn{2}{c|}{ L-NBee } & \multicolumn{2}{c|}{ L-UBee } & \multicolumn{2}{c|}{ Godunov2 } \\
\hline $1 / \Delta x$ & $\mathbf{L}^{1}$-err & $\gamma(\Delta x)$ & $\mathbf{L}^{1}$-err & $\gamma(\Delta x)$ & $\mathbf{L}^{1}$-err & $\gamma(\Delta x)$ & $\mathbf{L}^{1}$-err & $\gamma(\Delta x)$ & $\mathbf{L}^{1}$-err & $\gamma(\Delta x)$ \\
\hline 80 & $2.7 \mathrm{e}-02$ & - & $4.8 \mathrm{e}-02$ & - & $5.2 \mathrm{e}-03$ & - & $1.6 \mathrm{e}-02$ & - & $8.5 \mathrm{e}-03$ & - \\
\hline 160 & $1.9 \mathrm{e}-02$ & 0.53 & $3.4 \mathrm{e}-02$ & 0.52 & $2.9 \mathrm{e}-03$ & 0.83 & $5.8 \mathrm{e}-03$ & 1.5 & $5.5 \mathrm{e}-03$ & 0.64 \\
\hline 320 & $1.3 \mathrm{e}-02$ & 0.57 & $2.3 \mathrm{e}-02$ & 0.55 & $1.2 \mathrm{e}-03$ & 1.3 & $2.4 \mathrm{e}-03$ & 1.2 & $3.0 \mathrm{e}-03$ & 0.84 \\
\hline 640 & $8.6 \mathrm{e}-03$ & 0.58 & $1.6 \mathrm{e}-02$ & 0.57 & $5.1 \mathrm{e}-04$ & 1.3 & $1.4 \mathrm{e}-03$ & 0.74 & $1.7 \mathrm{e}-03$ & 0.87 \\
\hline 1280 & $5.7 \mathrm{e}-03$ & 0.59 & $1.0 \mathrm{e}-02$ & 0.58 & $3.6 \mathrm{e}-04$ & 0.51 & $9.4 \mathrm{e}-04$ & 0.63 & $8.0 \mathrm{e}-04$ & 1.0 \\
\hline
\end{tabular}

Table 3: Test 2. Non-local multi-class LWR model. Initial condition (4.3), with decreasing kernel functions, final time $T=0.5$. The reference solution is computed with $1 / \Delta x=5120$.

\subsection{Test 3. Autonomous and human-driven mixed traffic}

This test is intended to study how the presence of Connected Autonomous Vehicles (CAVs) impacts road traffic performances, as proposed in [10, Section 4.2]. Let us consider a circular road modeled by the space interval $[-1,1]$ with periodic boundary conditions at $x= \pm 1$. All 


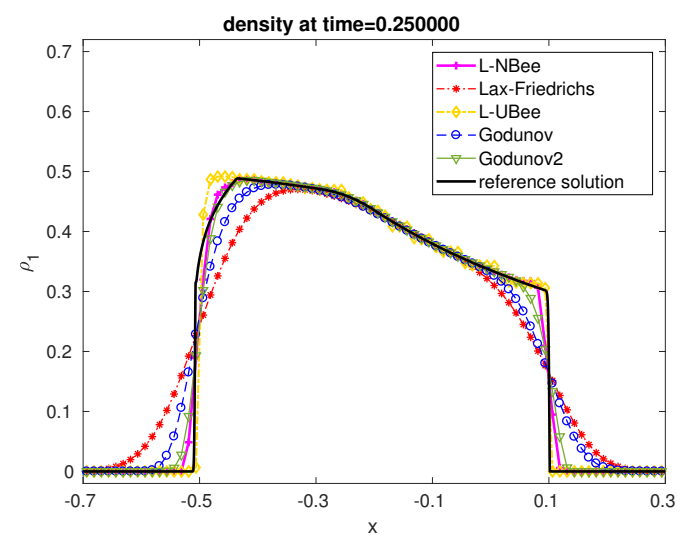

(a)

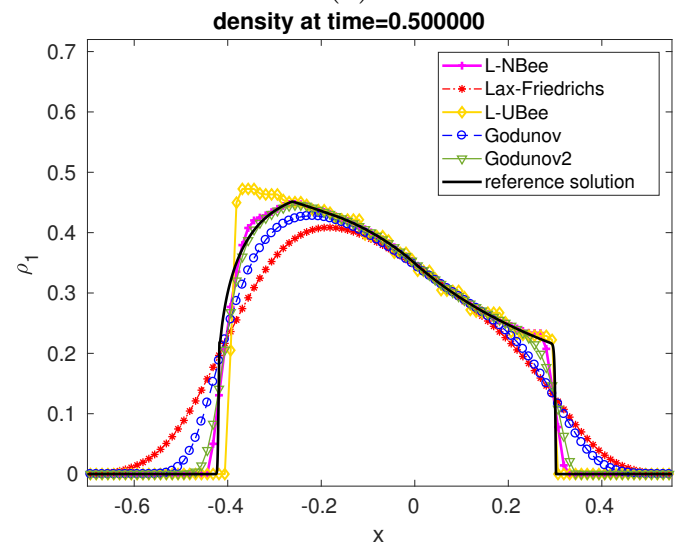

(c)

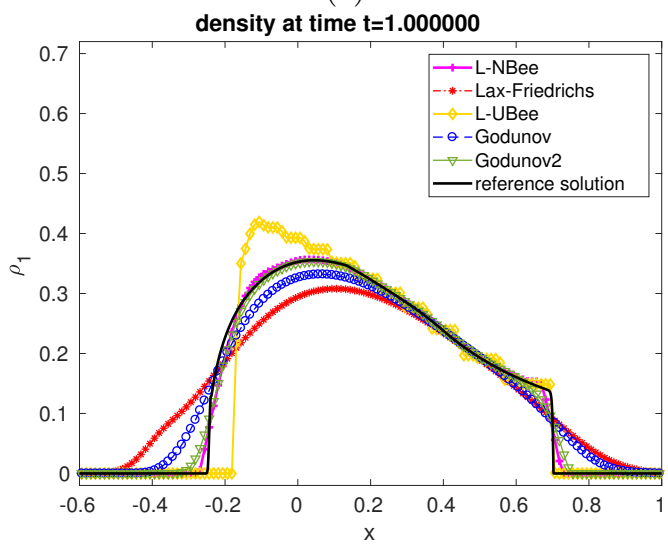

(e)

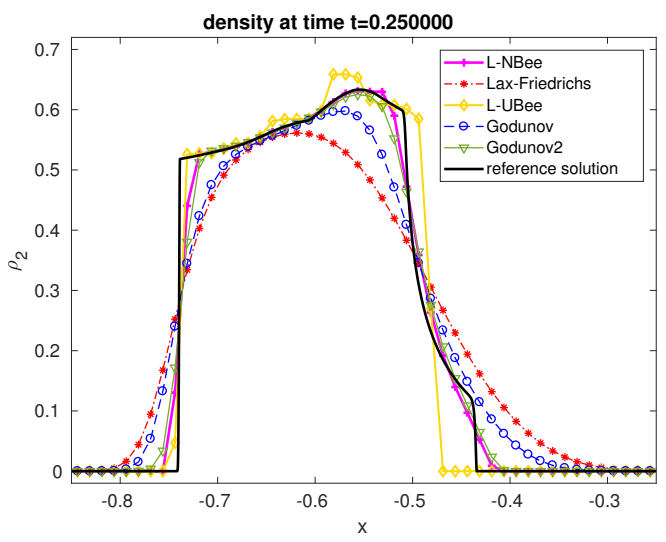

(b)

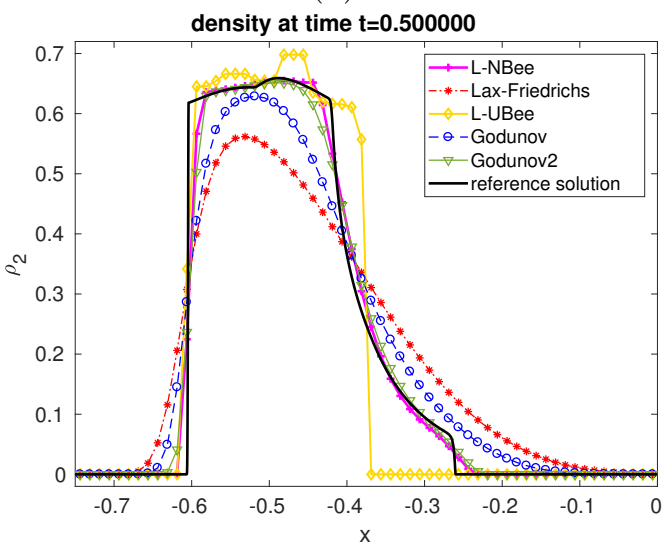

(d)

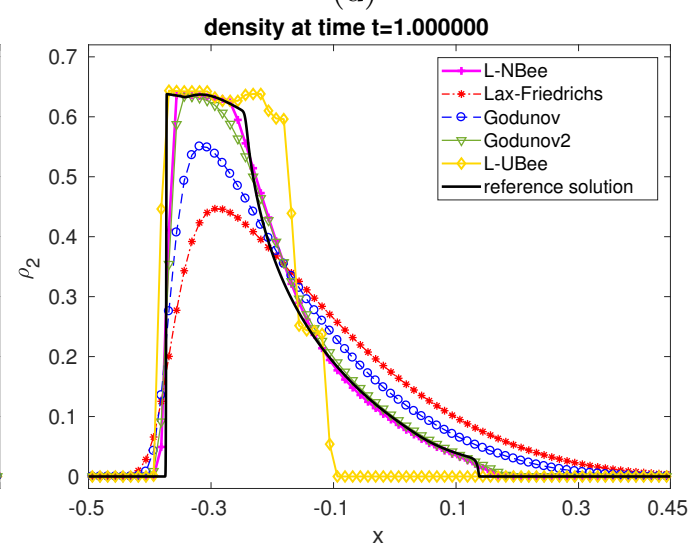

(f)

Figure 5: Test 2. (a)-(c)-(e) Profile of $\rho_{1}$ (trucks); (b)-(d)-(f) profile of $\rho_{2}$ (cars) computed with different numerical schemes at different times and $1 / \Delta x=80$.

the vehicles have the same maximal speed, but the interaction radius of CAVs is much grater than the one of human-driven cars. Moreover, we consider a constant convolution kernel to model the behaviour of CAVs, since the information they get about surrounding traffic is transmitted through wireless connections and it does not deteriorate with distance. We 


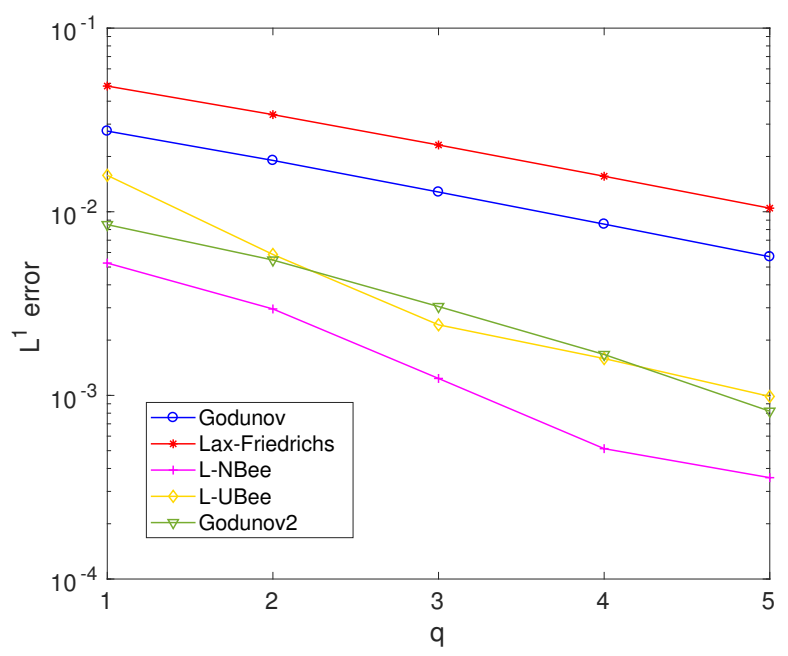

Figure 6: Test 2. Approximate total $\mathbf{L}^{\mathbf{1}}$-error for different numerical schemes with the decreasing kernel functions $\omega_{1}(x)=\omega_{2}(x)=2\left(\eta_{1,2}-x\right) / \eta_{1,2}^{2}, \eta_{1}=0.3, \eta_{2}=0.1$

consider the following initial data and parameters

$$
\begin{array}{ll}
\rho_{1}(0, x)=\beta(0.5+0.3 \sin (5 \pi x)), & \omega_{1}(x)=\frac{1}{\eta_{1}}, \quad \eta_{1}=1.0, \quad v_{1}^{\max }=1, \\
\rho_{2}(0, x)=(1-\beta)(0.5+0.3 \sin (5 \pi x)), & \omega_{2}(x)=\frac{2}{\eta_{2}}\left(1-\frac{x}{\eta_{2}}\right), \quad \eta_{2}=0.05, \quad v_{2}^{\max }=1,
\end{array}
$$

where $\rho_{1}$ is the density of autonomous vehicles and $\rho_{2}$ the density of human-driven vehicles. The parameter $\beta \in[0,1]$ gives the penetration rate of autonomous vehicle. Figure $7 \mathrm{a}$ displays the reference solution of (1.1)-(4.4) with $\beta=0.9$, computed by the second-order Godunov scheme with $1 / \Delta x=10240$ at time $T=1.5$. In Table 4 and Figure $7 \mathrm{~b}$ we compute the approximate $\mathbf{L}^{\mathbf{1}}$-error and the E.O.A at time $T=1.5$.

In Figure 8, we display separately the two classes and we compare the approximate solutions computed by all the considered schemes with $1 / \Delta x=320$, and the reference solution. Again, the numerical solutions obtained using the L-AR schemes are more anti-diffusive than those produced by first-order schemes. We observe a good behavior of the L-NBee scheme. On the other hand, the L-UBee scheme approaches the reference solution very well in the presence of shock-waves. On the other hand, the usual "stairs" appear in presence of rarefaction-waves.

We observe that the performances of L-NBee schemes are comparable with those of the second-order Godunov scheme. It is worth pointing out that despite the "staircaising" phenomenon the L-UBee $\mathbf{L}^{\mathbf{1}}$-error is still smaller than the $\mathbf{L}^{\mathbf{1}}$-error of the other first-order schemes.

\section{Conclusions}

We extended the L-AR schemes proposed in [5, 6] to the non-local multi-class traffic flow model proposed in [10. We provided some properties of the L-AR scheme and we proved the convergence to weak solutions in the scalar case. The proposed numerical tests indicate that these schemes are competitive with the first and second-order schemes proposed in the 


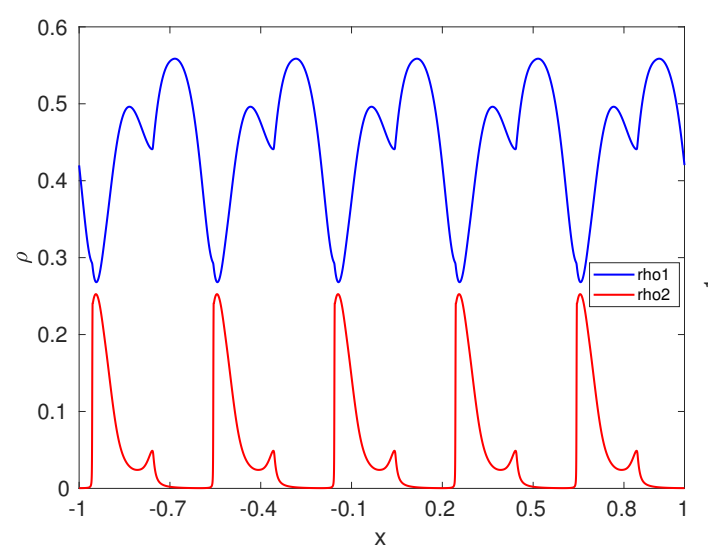

(a)

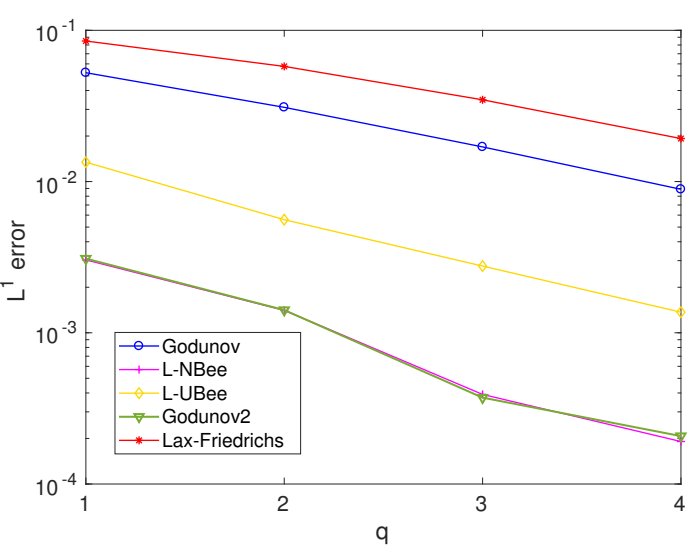

(b)

Figure 7: Test 3. (a) Reference solution of Test 3 at time $T=1.5$ computed with $1 / \Delta x=$ 10240. (b) Approximate total $\mathbf{L}^{\mathbf{1}}$-error for different numerical schemes.

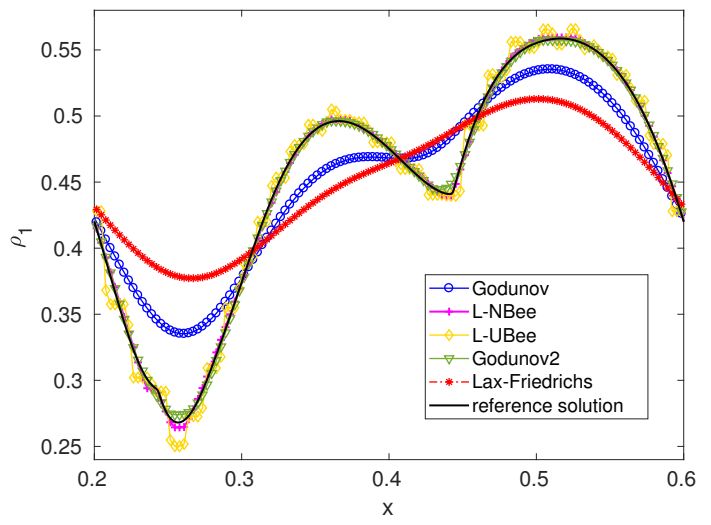

(a)

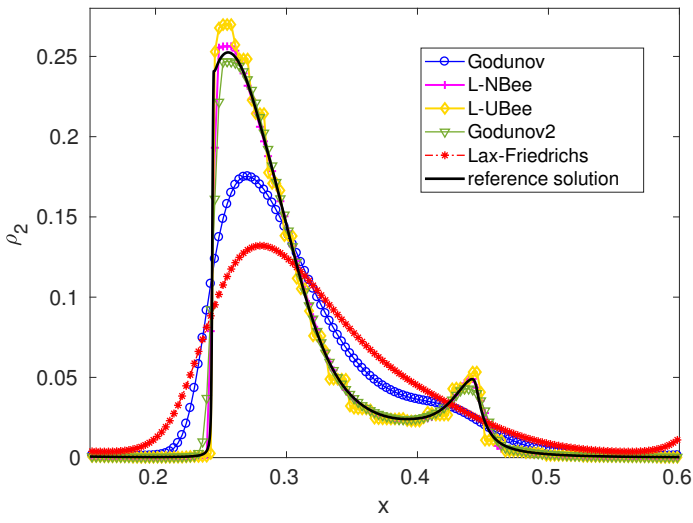

(b)

Figure 8: Test 3. (a) Profile of $\rho_{1}$; (b) profile of $\rho_{2}$, computed with different numerical schemes at time $T=1.5$ and $1 / \Delta x=320$. 


\begin{tabular}{|c|c|c|c|c|c|c|c|c|c|c|}
\hline & \multicolumn{2}{|c|}{ Godunov } & \multicolumn{2}{c|}{ Lax-F } & \multicolumn{2}{c|}{ L-NBee } & \multicolumn{2}{c|}{ L-UBee } & \multicolumn{2}{c|}{ Godunov2 } \\
\hline $1 / \Delta x$ & $\mathbf{L}^{1}$-err & $\gamma(\Delta x)$ & $\mathbf{L}^{1}$-err & $\gamma(\Delta x)$ & $\mathbf{L}^{1}$-err & $\gamma(\Delta x)$ & $\mathbf{L}^{1}$-err & $\gamma(\Delta x)$ & $\mathbf{L}^{1}$-err & $\gamma(\Delta x)$ \\
\hline 320 & $5.2 \mathrm{e}-02$ & - & $8.5 \mathrm{e}-02$ & - & $3.0 \mathrm{e}-03$ & - & $1.3 \mathrm{e}-02$ & - & $3.1 \mathrm{e}-03$ & - \\
\hline 640 & $3.1 \mathrm{e}-02$ & 0.76 & $5.8 \mathrm{e}-02$ & 0.56 & $1.4 \mathrm{e}-03$ & 1.1 & $5.7 \mathrm{e}-03$ & 1.3 & $1.4 \mathrm{e}-03$ & 1.1 \\
\hline 1280 & $1.7 \mathrm{e}-02$ & 0.87 & $3.5 \mathrm{e}-02$ & 0.73 & $3.9 \mathrm{e}-04$ & 1.8 & $2.8 \mathrm{e}-03$ & 1.0 & $3.7 \mathrm{e}-04$ & 1.9 \\
\hline 2560 & $8.9 \mathrm{e}-03$ & 0.93 & $1.9 \mathrm{e}-02$ & 0.85 & $1.9 \mathrm{e}-04$ & 1.0 & $1.4 \mathrm{e}-03$ & 1.0 & $2.0 \mathrm{e}-04$ & 0.84 \\
\hline
\end{tabular}

Table 4: Test 3. Initial condition (4.4), with different kernel functions, final time $T=1.5$. The solutions are computed with $1 / \Delta x=160 \times 2^{q}$ for $q=1, \ldots, 4$.

literature, in particular when more than one class are involved. If the initial datum has jump discontinuities, the performance of L-AR schemes are comparable with those of the secondorder Godunov scheme. The improvement to higher order of accuracy is an involved issue that can be studied in the near future.

\section{Acknowledgements}

This research was supported by the Inria Associated Team Efficient numerical schemes for non-local transport phenomena (NOLOCO; 2018-2020). LMV is supported by CONICYTChile through the project AFB170001 of the PIA Program: Concurso Apoyo a Centros Científicos y Tecnológicos de Excelencia con Financiamiento Basal; and by Centro de Investigación en Ingeniería Matemática $\left(\mathrm{CI}^{2} \mathrm{MA}\right)$, Universidad de Concepción; and Fondecyt-Chile project 1181511.

\section{References}

[1] A. Aggarwal, R. M. Colombo, and P. Goatin. Nonlocal systems of conservation laws in several space dimensions. SIAM J. Numer. Anal., 53(2):963-983, 2015.

[2] S. Benzoni-Gavage and R. M. Colombo. An n-populations model for traffic flow. European J. Appl. Math., 14(5):587-612, 2003.

[3] S. Blandin and P. Goatin. Well-posedness of a conservation law with non-local flux arising in traffic flow modeling. Numer. Math., 132(2):217-241, 2016.

[4] O. Bokanowski and H. Zidani. Anti-dissipative schemes for advection and application to Hamilton-Jacobi-Bellmann equations. J. Sci. Comput., 30(1):1-33, 2007.

[5] R. Bürger, C. Chalons, and L. M. Villada. Antidiffusive and random-sampling lagrangian-remap schemes for the multiclass Lighthill-Whitham-Richards traffic model. SIAM J. Sci. Comput., 35(6):B1341-B1368, 2013.

[6] R. Bürger, C. Chalons, and L. M. Villada. Antidiffusive Lagrangian-remap schemes for models of polydisperse sedimentation. Numer. Methods Partial Differential Equations, 32(4):1109-1136, 2016.

[7] R. Bürger, A. García, K. Karlsen, and J. Towers. A family of numerical schemes for kinematic flows with discontinuous flux. J. Engrg. Math., 60(3-4):387-425, 2008.

[8] C. Chalons, P. Goatin, and L. M. Villada. High-order numerical schemes for one-dimensional nonlocal conservation laws. SIAM J. Sci. Comput., 40(1):A288-A305, 2018.

[9] F. A. Chiarello and P. Goatin. Global entropy weak solutions for general non-local traffic flow models with anisotropic kernel. ESAIM: M2AN, 52(1):163-180, 2018. 
[10] F. A. Chiarello and P. Goatin. Non-local multi-class traffic flow models. Netw. Heterog. Media, 14(2):371 - 387, 2019.

[11] F. A. Chiarello, P. Goatin, and L. M. Villada. High-order Finite Volume WENO schemes for nonlocal multi-class traffic flow models. Proc. XVII International Conference on Hyperbolic Problems Theory, Numerics, Applications, 2019.

[12] B. Després and F. Lagoutière. Contact discontinuity capturing schemes for linear advection and compressible gas dynamics. J. Sci. Comput., 16(4):479-524, 2001.

[13] R. Eymard, T. Gallouët, and R. Herbin. Finite volume methods. In Handbook of numerical analysis, Vol. VII, Handb. Numer. Anal., VII, pages 713-1020. North-Holland, Amsterdam, 2000.

[14] J. Friedrich, O. Kolb, and S. Göttlich. A Godunov type scheme for a class of LWR traffic flow models with non-local flux. Netw. Heterog. Media, 13(4):531 - 547, 2018.

[15] P. Goatin and S. Scialanga. Well-posedness and finite volume approximations of the LWR traffic flow model with non-local velocity. Netw. Heterog. Media, 11(1):107-121, 2016.

[16] E. Godlewski and P.-A. Raviart. Numerical approximation of hyperbolic systems of conservation laws, volume 118 of Applied Mathematical Sciences. Springer-Verlag, New York, 1996.

[17] M. J. Lighthill and G. B. Whitham. On kinematic waves. II. A theory of traffic flow on long crowded roads. Proc. Roy. Soc. London. Ser. A., 229:317-345, 1955.

[18] P. I. Richards. Shock waves on the highway. Oper. Res., 4:42-51, 1956.

[19] P. K. Sweby. High resolution schemes using flux limiters for hyperbolic conservation laws. SIAM J. Numer. Anal., 21(5):995-1011, 1984.

[20] B. Van Leer. Towards the ultimate conservative difference scheme. v. a second-order sequel to Godunov's method. J. Comput. Phys., 32(1):101-136, 1979.

[21] J. von zur Gathen and J. Gerhard. Modern computer algebra. Cambridge University Press, Cambridge, second edition, 2003. 